Cahiers de recherches médiévales

\title{
Variations, vie et mort des Loherains
}

Réflexions sur la gestation et les paradoxes d'un grand cycle épique

Jean-Charles Herbin

\section{CpenEdition}

Journals

Édition électronique

URL : https://journals.openedition.org/crm/2242

DOI : $10.4000 / \mathrm{crm} .2242$

ISSN : 1955-2424

Éditeur

Honoré Champion

\section{Édition imprimée}

Date de publication : 30 décembre 2005

Pagination : 147-174

ISSN : $1272-9752$

\section{Référence électronique}

Jean-Charles Herbin, «Variations, vie et mort des Loherains », Cahiers de recherches médiévales [En ligne], 12 | 2005, mis en ligne le 30 décembre 2008, consulté le 15 décembre 2022. URL : http:// journals.openedition.org/crm/2242; DOI : https://doi.org/10.4000/crm.2242

Ce document a été généré automatiquement le 15 décembre 2022.

Tous droits réservés 


\section{Variations, vie et mort des Loherains}

Réflexions sur la gestation et les paradoxes d'un grand cycle épique*

Jean-Charles Herbin

\section{La Geste des Loherains : une appellation trompeuse}

1 Lorsqu'il livre au public, en 1833, la première édition moderne de la Chanson de Garin, Paulin Paris l'envisage comme «faisant partie d'un autre poème plus vaste, désigné sous le nom général de Chanson des Lohérens ". À l'époque, il conçoit l'ensemble de manière généalogique, mêlant (par l'usage approximatif, pour nous, des italiques et des caractères romains), les noms des personnages et les titres des poèmes : «Les Lohérens comprennent les histoires $-1^{\circ}$ du duc Hervis de Mez; $-2^{\circ}$ de Garin le Loherenc et Begon de Belin, ses fils ; $-3^{\circ}$ de Girbert, fils de Garin, Hernaut et Girbert, fils de Bégon ; $-4^{\circ}$ enfin d'une quatrième génération que les continuateurs ont poursuivie jusqu'au célèbre Garin de Monglave (sic) $»^{1}$. Dès 1846, dans la préface qu'il donne à sa Mort de Garin le Loherain, Édélestand du Méril utilise, pour désigner ce qu'il considère comme un cycle, l'appellation Geste des Lorrains, promise à un bel avenir ${ }^{2}$, mais qui ne sera jamais définie avec précision et qui recouvre, au fond, plus un fourre-tout qu'un cycle clairement défini.

2 En 1863, Paulin Paris précise sa pensée: «Qu'est-ce que la Geste des Loherains, se demande-t-il, cette longue chanson dont le Garin forme la première partie ? $»^{3}$. Et de poursuivre : «Je ferais peut-être bien de répondre comme l'auteur de la rédaction en prose du Saint Graal : "C'est l'histoire tirée de toutes les histoires" »". Quant au rapport des grandes figures de la chanson avec celles de «l'histoire authentique», «...j'ai torturé si longtemps mon esprit à de vaines recherches, avouait le vénérable médiéviste, que j'ai bien le droit de jeter, comme on dit, ma langue à de plus jeunes barbes $»^{5}$.

3 Quoi qu'il en soit, sa vision d'ensemble est restée confuse. Tout au plus comprend-on qu'en 1863 Paulin Paris envisageait la Geste des Loherains non plus comme une suite de récits parallèles aux générations des héros, mais comme un ensemble constitué de deux 
parties ${ }^{6}$, alors que dans la synthèse qu'il avait signée dans l'Histoire littéraire de la France, il concluait en distinguant trois parties attribuables à des auteurs différents : Hervis (auteur anonyme), Garin/Gerbert (Jehan de Flagy, les limites du texte restant approximatives), une « dernière partie » (due à plusieurs trouvères écrivant en rimes)7

C'est sur cette division floue et insuffisamment interrogée qu'a tourné, depuis lors, sans remise en cause fondamentale ${ }^{8}$, l'essentiel du discours critique sur la Geste des Loherains, qu'aucun manuscrit médiéval - le constat est à souligner - ne nous a transmise avec ce titre et en intégralité. Il est peut-être temps de réexaminer le bien-fondé de cette conception ancienne. Nous avons aujourd'hui la chance d'être loin des querelles postromantiques et nationalistes qui voulaient, à la fin du XIX et au début du $\mathrm{XX}^{\mathrm{e}}$ siècle, que les chansons de geste soient françaises d'abord pour ne pas être germaniques et, par voie de conséquence, qu'il y ait eu une "geste lorraine ", et donc française, qui montrait, pensait-on et pour faire court, qu'au Moyen Âge les Lorrains avaient déjà choisi la culture française.

5 Comme nous l'avons montré ailleurs', les chansons d'Anseÿs de Gascogne (et non plus de Metz) et de la Vengeance Fromondin doivent être mises à part du cycle, dont elles ne font que compromettre l'unité. Il y avait bien, au début du XIV ${ }^{e}$ siècle, dans l'esprit des contemporains du succès de nos héros épiques, deux cycles séparés : les viés Loherens et les novials, pour reprendre la distinction utilisée dans l'inventaire des livres du comte Guillaume III de Hollande, alias Guillaume $\mathrm{I}^{\mathrm{er}}$ de Hainaut ${ }^{10}$. Il est clair pour nous que le premier cycle, qui seul mérite l'appellation de Geste des Loherains, se composait de Garin et Gerbert, et très vraisemblablement à l'époque de l'inventaire considéré, de Hervis et d'Yonnet $^{11}$. Ces quatre poèmes représentent ce que nous appellerions volontiers le "Grand Cycle » des Loherains, l'ensemble offrant une belle cohérence thématique (sauf peut-être Hervis, plus romanesque), une continuité généalogique sans faille - ce qui ne veut pas dire sans difficultés ${ }^{12}$ - et une conclusion lorraine.

6 Parmi les « Lorrains nouveaux », nous rangeons sans hésiter Anseÿs de Gascogne, et peutêtre la Vengeance Fromondin, dont l'intention, toutefois, est radicalement différente et même opposée. Ce second cycle des Loherains, beaucoup plus récent que le premier (puisqu'il ne date, au plus tôt, que des années $1250-1260)^{13}$, n'est pas du tout dans l'esprit du premier cycle, dont il se sert pour régler des comptes qui n'ont plus rien à voir avec les enjeux primitifs : en bref, l'hostilité du lignage bordelais et du lignage lorrain, hostilité qui constituait le moteur de la véritable Geste des Loherains, se trouve, dans ces deux poèmes, mise à contribution pour régler les comptes des seigneurs du Nord et du roi de France (Anseÿs), du roi de France et du comte de Boulogne (Vengeance Fromondin). En définitive, seul l'énorme succès du Cycle originel ${ }^{14} \mathrm{a}$ pu justifier cette « récupération » et la fabrication sur mesure (Anseÿs et la Vengeance Fromondin sont, très vraisemblablement, des œuvres de clercs, ne reprenant aucune tradition orale préexistante) d'un second cycle après 1250-1260, second cycle qui doit être regardé non pas comme un prolongement sincère, mais essentiellement comme un éclatement $d u$ "Grand Cycle » qui lui préexiste.

$7 \quad$ Mais il nous faut encore affiner notre estimation, l'existence des deux entités cycliques évoquées précédemment ne constituant qu'une considération valable pour le début du $\mathrm{XIV}^{\mathrm{e}}$ siècle. Revenons à la véritable Geste des Loherains, le premier (Grand) Cycle défini plus haut: Garin / Gerbert, Hervis, Yonnet.

8 Il est clair pour nous que le cycle primitif ne devait compter que Garin et Gerbert, que la tradition nous a presque toujours transmis ensemble ${ }^{15}$ : comptant plus de trente mille 
vers au total, ces deux poèmes étaient déjà en mesure de constituer un cycle, d'autant plus qu'avant la présentation actuelle du texte dans les manuscrits, qui permet d'envisager séparément un Garin et un Gerbert - en accord avec la présence d'une très grande initiale à la jointure supposée dans la plupart des témoins parvenus ${ }^{16}-$, une autre distinction avait prévalu, qui faisait de cet ensemble le regroupement non pas de deux, mais de trois entités: Garin I, Garin II, Garin III, pour reprendre le découpage retenu par Philippe de Vigneulles, qui ne l'a pas inventé ${ }^{17}$. En bref, Garin I correspond aux v. 1-9549 de l'édition Iker-Gittleman, Garin II aux v. 9550-15950 environ; quant à Garin III, il englobe les 2700 derniers vers de l'édition de Garin le Loherain et tout le texte du Gerbert (en fait, dans sa version longue encore inédite) ${ }^{18}$. Pour être tout à fait précis, ajoutons dès maintenant que le vrai noyau de l'ensemble paraît bien être Garin II, qui s'ouvre par l'épisode de la mort de Bégon. Philippe Mousket, qui écrivait avant 1243, conforte cette hypothèse : il ne fait état que de données tirées de Garin II et Garin III ; il accorde, qui plus est, une place disproportionnée à l'épisode précis de la mort de Bégon (36 vers, 2080-2125), réduisant tout le reste à une vingtaine de vers (2126-2145). On a remarqué depuis longtemps que Philippe Mousket ignorait totalement Hervis, Anseÿs et la Vengeance Fromondin (ces deux derniers poèmes étant à peu près certainement postérieurs à l'époque de composition de la Chronique rimée) ; il n'est peut-être pas inutile de souligner que Philippe Mousket ignore tout autant le contenu de Garin I, notamment tout le Prologue consacré aux exploits du duc Hervis. De surcroît, c'est seulement, selon nous, lors d'une seconde étape d'expansion du cycle primitif - vers ce que nous avons appelé le "Grand Cycle» - qu'il faut prendre en considération la composition du poème de Hervis (vers 1200-1210) et d'Yonnet (difficile à dater, mais du XIII ${ }^{e}$ siècle), poèmes qui sont venus compléter le cycle primitif par expansion généalogique ascendante, pour le premier, descendante, pour le second. Avant de nous interroger plus précisément sur ses différents éléments, voici donc comment nous concevons désormais ce qu'on appelait peut-être un peu trop vite la Geste des Loherains :

Geste des Loherains proprement dite (ou « Grand Cycle des Lorrains »):

- Garin/Gerbert (Cycle primitif)

- Hervis/Yonnet (Expansion en cohérence avec le Cycle primitif)

Pseudo-Geste des Loherains:

- Anseÿs de Gascogne

- Vengeance Fromondin

9 Il faut encore, en préliminaire, dégager le fonctionnement de l'ensemble de la Geste proprement dite: la matière paraît s'être organisée par strates et remaniements successifs autour d'un noyau ancien. Dans ce qui suit, nous faisons état, sous forme d'hypothèses, des convictions que nous avons pu asseoir solidement, nous l'espérons, pour certaines d'entre elles, depuis plus de vingt ans que nous menons nos recherches sur les Loherains; mais, à l'occasion, nous n'hésiterons pas à proposer quelques perspectives que nous n'avons pas encore pu examiner jusque dans les détails ${ }^{19}$. Nous espérons rendre compte ainsi d'une manière cohérente et convaincante de l'évolution de la matière épique considérée.

10 Après cette mise au point nécessaire, nous pouvons reprendre un par un les divers éléments que l'on peut individualiser dans la Geste, à commencer par ce que nous considérons comme le noyau de celle-ci. 


\section{Hypothèse 1 . Un noyau très ancien: la Mort Begon (non lorrain)}

11 Nous remettons en cause, dans cette première rubrique, non pas la datation du poème de Garin au XII ${ }^{\mathrm{e}}$ siècle, datation traditionnellement admise, mais celle d'un de ses composants, la Mort Begon. Pour ce faire, nous considérons qu'il y a bien, à l'origine, un événement authentique, probablement le meurtre d'un grand personnage, qu'il se soit appelé Bégon ou autrement. Sinon, on comprendrait mal pourquoi le poète se serait astreint à un carcan "réaliste" comme celui que nous allons examiner rapidement maintenant, et qui concerne surtout l'épisode de la mort de Bégon.

Le meurtre du duc Bégon lance réellement la guerre inexpiable entre les Loherains et les Bordelais ; jusque-là, il ne s'agissait que d'une concurrence - féroce et volontiers violente, il est vrai - entre deux puissants lignages féodaux. C'est probablement parce qu'il en avait compris l'importance qu'Edward Le Glay traduisit et publia séparément l'épisode dès $1835^{20}$ (cinq ans avant la "résurrection" du Roland d'Oxford...) : c'est là une des plus belles pages épiques françaises ${ }^{21}$. Mais il y a plus : c'est probablement aussi l'une des plus anciennes, sinon la plus ancienne. On nous pardonnera de ne faire qu'évoquer ou reprendre ici rapidement nos principaux arguments plaidant en ce sens $^{22}$. Un certain nombre de traits suggèrent fortement la grande ancienneté de l'épisode, même s'il paraît nous être parvenu en partie délabré. Ainsi, le héros, partant de "Gaudemont", c'est-à-dire Gademont, lieu-dit situé sur le territoire de Raismes (face à Valenciennes, de l'autre côté de l'Escaut) ${ }^{23}$ et se dirigeant vers l'Ouest, ne peut pas ignorer l'abbaye de Vicoigne, sauf, bien sûr, si elle n'existe pas: or, l'abbaye a été instituée en 1132 ; de plus, on aurait du mal à croire qu'un sanglier qui ravage la région ait pu élire son gîte à un jet de pierre de l'abbaye sans être inquiété.

13 En outre, l'abbé Lietri de Saint-Amand dans l'épisode renvoie, à l'évidence, à Liederic, abbé d'Elnon entre 952 et 956 . On nous rétorquera peut-être que le poète a pu trouver ce nom dans les archives de l'abbaye : soit. Toutefois, toutes les archives de l'Abbaye de Saint-Amand disparurent dans les flammes lors du grand incendie de 1066, ce qui inviterait à remonter assez haut la "fabrication" de l'épisode. Mais, même dans ce cas, il y aurait bien eu un événement authentique à la base du récit épique, sinon, pourquoi arrêter son choix sur cet abbé plutôt que sur un autre? Les noms mêmes de Liederic et de sa mère Liétarde, en effet, ne peuvent pas ne pas faire penser aux prénoms Liétard/ Liutard, qui, à la fin du IX et au début du $\mathrm{X}^{\mathrm{e}}$ siècle, paraissent attachés au comté de Metz et à la famille du seul Bégon historique de quelque importance $(\dagger 816)^{24}$. Ce sont là des rencontres troublantes qu'on ne peut négliger. En outre, il doit y avoir eu une première mise en forme écrite ou orale du récit de l'événement: sinon, pourquoi un poète se serait-il imposé, comme on va le voir, un cadre "réaliste" en contradiction avec l'expérience de son public?

En effet, en poursuivant le sanglier, Bégon ne traverse aucun cours d'eau qui se présenterait sur sa route: or, il suffit de regarder une carte pour s'apercevoir que le périple de Bégon et du sanglier entre Raismes et les bois de Lens oblige à traverser la Scarpe. Le texte de la Vulgate, c'est-à-dire, pour ce passage, la totalité des manuscrits de Garin le Loherain (ce que nous avons appelé plus haut le "Cycle primitif des Lorrains ») ${ }^{25}$, présenterait donc une sérieuse contradiction avec le paysage réel : à quoi bon nous avoir dit que Bégon traverse la Gironde (v. 9638), la Somme à Cléry (v. 9652) et l'Escaut (v. 9713), à quoi bon localiser l'action avec la précision des (micro)toponymes (Raismes 
v. 9719 , Gaudemont v. 9752) ${ }^{26}$, si c'est pour se montrer si approximatif sur le terrain de la chasse tragique ? À moins d'admettre chez l'auteur de Garin le Loherain, si l'on refuse notre hypothèse d'un noyau très ancien réutilisé beaucoup plus tard, une démarche "réaliste" totalement inconnue dans la littérature et l'esprit épiques, consistant à adjoindre au récit des éléments réalistes "archaïques" que seul un auteur désireux de faire œuvre de faussaire pouvait connaître à la fin du XII siècle. Il convient donc d'examiner le texte de plus près.

En fait, il n'est pas dit que Bégon traverse un cours d'eau, mais que ses chevaliers, dès qu'il les a distancés, s'embourbent à qui mieux mieux, contraints finalement de renoncer à suivre la chasse (v. 9760-9768). Le terrain est donc très marécageux. Si Bégon échappe aux pièges de la boue et des fondrières, c'est sans doute qu'il suit des yeux son sanglier et qu'il ne le perd jamais de vue : or, le sanglier connaît les lieux et son instinct, de toute façon, doit lui faire trouver tout naturellement les gués ou les endroits les plus praticables. C'est ici qu'il convient de rappeler que la Scarpe n'est, au fond, qu'une vallée marécageuse, dans laquelle, à l'origine, serpente une infinité de bras secondaires. Avant les travaux initiés par le comte de Flandre Arnoul le Grand († 964), avant le détournement de la Sensée, destiné à rendre la Scarpe navigable, cette dernière, pour dire les choses sans ambages, n'existe pas. En 952-956 (abbatiat de Liederic de Saint-Amand), la situation est donc bien celle qui est décrite dans la Vulgate : une vallée marécageuse, pas de cours d'eau principal faisant obstacle.

Cette argumentation peut paraître spécieuse : pourtant, le remanieur à qui nous devons la famille INT s'est rendu compte du problème bien avant nous. De son temps, les travaux devaient avoir produit un changement de paysage tel que le public local ne pouvait plus admettre la leçon primitive. La Scarpe, grossie des eaux de la Sensée, surcreusée et entravée de moulins, était devenue, à n'en point douter, une véritable rivière, qu'on n'aurait pu ignorer et franchir sans s'en apercevoir ${ }^{27}$. En faisant traverser l'Escaut à Bégon plus au Nord et en situant le gîte du sanglier "delez Landas ", c'est-àdire près de Landat, localité située sur la rive gauche de la Scarpe, le remanieur de la famille INT règle la difficulté topographique, et Bégon peut s'élancer vers les bois de Lens qui se trouvent à quinze lieues de là, sans rencontrer de véritable cours d'eau sur son chemin.

L'épisode de la mort de Bégon n'a, à vrai dire, rien de spécifiquement lorrain, à moins de rappeler qu'en quittant Valenciennes et en traversant l'Escaut, le duc sort de Lorraine, c'est-à-dire du royaume de Lothaire, compris entre le Rhin à l'Est et l'Escaut à l'Ouest, tel qu'il fut précisément défini, après le Traité de Verdun de 843 et avant 855, par Lothaire $\mathrm{I}^{\mathrm{er}}$ en faveur de son fils Lothaire $\mathrm{II}^{28}$. En vérité, cet épisode ne trouve vraiment sa cohérence géographique qu'à une époque qu'on ne peut guère situer plus bas que le milieu ou le début du troisième quart du $\mathrm{X}^{\mathrm{e}}$ siècle, c'est-à-dire juste avant les travaux qui allaient, dès la fin du siècle, modifier le paysage réel au point que la version primitive deviendrait (au moins localement) inacceptable. Si l'on admet qu'une génération, deux tout au plus, sont susceptibles d'avoir conservé le souvenir de l'événement sous une forme orale, on doit pouvoir dater la Mort Begon originelle, noyau des Loherains, de la fin du $\mathrm{X}^{\mathrm{e}}$ siècle, au plus tard (ou, en tout état de cause, avant l'incendie de 1066, si la première origine écrite reposait sur un document figurant dans les archives de l'Abbaye de Saint-Amand). C'est à peu près l'époque où les derniers descendants de Charlemagne perdent le pouvoir, au profit de la famille capétienne. Cette première conclusion permet, en outre, de dégager la famille INT et de la 
considérer comme un remaniement postérieur à la constitution de la Vulgate, ellemême présentant la première intégration de l'épisode de la mort de Bégon dans ce qu'il convient d'appeler le «Cycle primitif» des Loherains.

Combien de temps, si notre hypothèse se tient, la Mort Begon est-elle restée une œuvre isolée, nous l'ignorons. Il a pu y avoir maintien des choses en l'état, maintien d'une tradition locale pendant les $\mathrm{XI}^{\mathrm{e}}$ et $\mathrm{XII}^{\mathrm{e}}$ siècles, jusqu'au moment où un poète, Jean de Flagy ou un autre, aura mis en ordre la matière de Garin, telle que nous la connaissons.

\section{Hypothèse 2. Une lente maturation ou une mutation brusque au cours des $\mathrm{XI}^{\mathrm{e}}-\mathrm{XII}{ }^{\mathrm{e}}$ siècles?}

Dans cette deuxième rubrique, nous reprenons, grosso modo, les conclusions admises par la plupart des médiévistes concernant la gestation et l'évolution des Loherains aux $\mathrm{XI}^{\mathrm{e}}$ $\mathrm{XII}^{\mathrm{e}}$ siècles. C'est, en fait, essentiellement durant le XII ${ }^{e}$ siècle que l'on s'accorde à situer la mise en forme de ce que, plus haut, nous avons appelé la Vulgate ou le «Cycle primitif ». Encore faut-il nuancer et distinguer, une fois de plus.

Les dates retenues par les uns et les autres pour Garin le Loherain varient cependant considérablement à l'intérieur du $\mathrm{XII}^{\mathrm{e}}$ siècle $^{29}$. Mais on doit pouvoir accorder les avis divergents en retenant l'idée que le cycle que constituent Garin I et II n'est pas sorti tout armé de l'esprit d'un seul poète. On a identifié depuis longtemps un groupe d'une vingtaine de personnages historiques de la fin $\mathrm{du} \mathrm{XI}^{\mathrm{e}}$ ou du début du XII ${ }^{\mathrm{e}}$ siècle, qui apparaissent avec leurs noms et leurs titres dans la fiction ${ }^{30}$. On ne s'est probablement pas assez inquiété du fait que ces personnages se présentent le plus souvent groupés dans des listes, et seulement dans quelques épisodes, ce qui suggère des strates dans la composition, plutôt qu'une composition en une seule fois. À vrai dire, ces personnages, pour reconnaissables qu'ils soient, ne sont en général que des comparses, que l'on a dû intégrer dans le récit pour l'ancrer, par cet artifice, dans la réalité historique, à moins que ce ne soit pour flatter ou malmener tel ou tel puissant personnage ou lignage. De plus, sur les vingt-sept personnages identifiés par J. Zezula dans sa récapitulation ${ }^{31}$, on note dix Loherains parmi les treize personnages les plus anciens ( $\mathrm{IX}^{\mathrm{e}}$-premier quart $\mathrm{du}$ $\mathrm{XI}^{\mathrm{e}}$ siècle), mais à l'inverse onze des quinze personnages les plus récents sont des Bordelais (deuxième quart du $\mathrm{XI}^{\mathrm{e}}$-première moitié du XII ${ }^{\mathrm{e}}$ siècle) : même si la raison nous en échappe, cela a bien dû avoir un sens dans les intentions du poète ou, pour mieux prendre en compte la gestation de ce qui constitue pour nous la Vulgate actuelle, les intentions des auteurs successifs, et cette situation suggère au moins deux grandes étapes pour la constitution de cette Vulgate.

Quoi qu'il en soit, ces personnages peuvent aussi nous éclairer sur la date de la mise en forme de la Vulgate, si nous parvenons à identifier les plus récents. Pour notre part, nous retiendrons que Begon compte parmi ses parents et alliés un Tiebaut d'Aspremont, seigneur de Dun (v. 4567-4571): or, l'Histoire nous apprend que cette conjonction de nom et de titre ne se produit pas avant Gobert IV d'Aspremont († 1162), qui avait obtenu Dun par son mariage. Plus intéressant, parce que permettant de resserrer encore la chronologie, l'archevêque Henri. Dans Garin, l'archevêque Henri de Rains ne peut avoir pour modèle que Henri de France, archevêque historique de Reims, et frère du roi Louis VII ; le fait que l'archevêque de Reims joue un rôle peu reluisant dans le poème - il refuse de participer financièrement à la défense du royaume inciterait à chercher le poète à qui on doit le Prologue et, probablement, la première 
mise en forme de ce que nous appelons le «Cycle primitif », ailleurs que parmi les amis de la famille capétienne ${ }^{32}$.

Cette dernière information fournirait un terminus tout à fait acceptable pour la dernière mise en forme de la matière léguée par un siècle au moins de gestation et de remaniements. C'est cette ultime mise en forme de la Vulgate que nous attribuerions volontiers à ce Jean de Flagy dont le nom se lit après le dernier vers de Garin le Loherain dans les manuscrits EMPF. Qu'il ait inventé tout ou partie de sa matière ou qu'il ait plutôt, comme nous le pensons, seulement organisé une matière éparse en cousant entre eux des éléments à l'origine disparates, c'est cependant ce que l'on ne peut pas trancher en toute certitude. On ignore donc la nature exacte et les limites de l'intervention de ce Jean de Flagy, comme on ignore l'époque où il a vécu ${ }^{33}$. Cependant, on peut légitimement penser que la conservation de son nom dans plusieurs témoins fait plus que suggérer une simple participation à la tradition de l'œuvre. Nous voulons croire que c'est lui qui a donné sa forme actuelle à la Vulgate.

Le cas de Gerbert est différent, sans être plus simple. On s'accorde en général à le dater entre 1185 et $1210^{34}$, mais ce choix repose surtout sur le fait qu'on y voit une chanson moins sauvage et plus romanesque que Garin, d'où l'on voudrait inférer une date de composition plus basse. Il est vrai que Gerbert n'a pas le caractère sombre et tragique de Garin, mais nous ne pensons pas pouvoir le dater par rapport à ce dernier en nous appuyant sur son contenu ou son style. Gerbert contient des éléments visiblement plus anciens que la fin du XII siècle, à commencer par l'épisode du crâne de Fromont, transposition de la légende de Cunnimond, tirée de l'Historia Longobardorum de Paul Diacre à une époque où, vraisemblablement, l'on comprenait encore le sens des divers éléments composant les anthroponymes d'origine germanique ${ }^{35}$. Gerbert, que peu de chose, au fond, sauf la généalogie de son héros éponyme, rattache à la Lorraine, a pu coexister avec Garin avant qu'un remanieur (Jean de Flagy ?) ne travaille à relier les deux poèmes, avec, en aval de la tradition, l'accident étrange - une transition qui paraît bégayer - que signale Félix Lecoy dans son article de $1956^{36}$.

À la fin du XII ${ }^{\mathrm{e}}$ siècle, malgré quelques divergences parfois importantes (familles NT, QS, notamment $)^{37}$, la Vulgate de Garin/Gerbert, devait se présenter grosso modo sous la forme que nous lui connaissons d'après les témoins parvenus et avec le contrôle qu'on peut en faire à l'aide des énumérations qui figurent à la fin de certains manuscrits ${ }^{38}$. On peut donc considérer qu'au tournant des $\mathrm{XII}^{\mathrm{e}}$ et $\mathrm{XIII}^{\mathrm{e}}$ siècles, était constitué ce que nous avons appelé plus haut le «Cycle primitif des Lorrains ${ }^{39}$, car c'est bien l'existence de ce premier cycle qui explique les expansions ascendante et descendante qui vont, au XIII ${ }^{e}$ siècle, constituer la Geste des Loherains proprement dite.

\section{Hypothèse 3. Le « Grand Cycle des Loherains " au complet (premières décennies du XIII e siècle - "enracinement" lorrain)}

Dans cette rubrique, nous reprendrons d'abord les résultats de nos précédents travaux sur le poème de Hervis.

D'après l'état des alliances que l'on constate dans l'épisode (remanié) du Tournoi de Senlis ${ }^{40}$, la composition de Hervis de Metz doit pouvoir être datée, au plus tard, du milieu de la seconde décennie du XIII siècle, mais rien ne s'oppose à ce qu'on la remonte autour des années $1200^{41}$. L'existence du premier cycle examiné dans ce qui précède appelait, à Metz en particulier, une expansion cyclique ascendante. Mais il convient de 
constater que ce prolongement n'est plus vraiment dans l'esprit des deux premiers composants du cycle: Hervis est une chanson d'aventures plus qu'une véritable chanson de geste ; de plus, dans ce texte, les Bordelais sont totalement absents, les enjeux étant radicalement étrangers à ceux de Garin et Gerbert. La revendication du narrateur à l'ouverture de la version $N$ du poème (« Mais j'en dirai, que bien l'ai espiié Toute l'estoire qu'a Mes est remenbré ») n'est sans doute pas une parole vaine. De fait, le poème de Hervis est infiniment plus lorrain, messin en vérité, que les textes auxquels il vient donner une ascendance, sans toutefois présenter avec eux de contradictions inacceptables ${ }^{42}$. Mais son intégration dans le cycle s'est faite en deux temps: dans le manuscrit $E$, le plus ancien $\left(\mathrm{XIII}^{2}\right)$, par simple juxtaposition ${ }^{43}$ avec Garin/Gerbert; puis dans NT (début du $\mathrm{XIV}^{\mathrm{e}}$ siècle au plus tard), par l'adjonction d'une sorte de transition de 1500 vers environ ${ }^{44}$ : ce mode opératoire d'intégration progressive a probablement été commun à Hervis et aux précédentes avancées dans la constitution d'un véritable cycle des Lorrains.

Dans la même perspective, il fallait une conclusion lorraine aux Loherains. C'est chose faite avec Yonnet de Metz. Ce poème n'est parvenu jusqu'à nous, sous une forme versifiée, que défiguré dans la rédaction $N$, où il sert de pré-texte à une version écourtée d'Anseÿs. Heureusement, nous disposons de la mise en prose de Philippe de Vigneulles (datée de 1515), et le travail du chaussetier messin, dont nous préparons une édition ${ }^{45}$, a sauvé de l'oubli un texte qui donne tout son sens à la notion de "Grand Cycle des Lorrains"».

En effet, dans Yonnet, tout se termine à Metz. Les personnages paraissent mettre un soin tout particulier à refaire à l'envers le chemin des premiers héros: dans Garin, les Loherains progressent vers l'Aquitaine, avec les guerres interminables qui en découlent ; dans Yonnet, un des derniers Loherains installés en Gascogne, le roi Gerbert, vient se faire tuer à Lens, fief originel des Bordelais. C'était déjà le chemin suivi par le duc Bégon qui, de Belin-Beliet en Aquitaine, venait chercher la mort dans les bois de Lens.

On peut estimer que Yonnet de Metz, qui devait compter environ 2250 vers, représente la seule conclusion interne de la Geste des Loherains proprement dite, qu'elle institue en cycle fermé définitivement: Hervis de Metz, Garin I, II, III et Yonnet de Metz. La "vraie" Geste se réduit donc aux cinq textes cités, dont le premier (Hervis) et le dernier (Yonnet) font de Metz le point de départ et d'arrivée du «Cycle » qu'ils constituent par leur existence même. Si ce «Grand Cycle des Lorrains » est cohérent, ou mieux, s'il en existe un état cohérent, c'est bien celui que propose l'existence du Yonnet.

\section{Hypothèse 4. L'éclatement (enjeux non lorrains)}

La place de la Vengeance Fromondin et du poème d'Anseÿs de Gascogne, que l'on considère en général comme deux des trois fins possibles de la Geste des Loherains (avec Yonnet de Metz), est problématique ${ }^{46}$.

31 Pour Anseÿs de Gascogne, l'affaire est entendue ${ }^{47}$, car c'est une œuvre assurément d'inspiration flamande: vocables flamands, traits dialectaux repérables dans les assonances, idéologie flamandophile enfin, qui en fait un immense poème à la gloire des féodaux du nord du royaume de France, et plus précisément d'un comte de Flandre imaginaire, Bauche li Cors, parangon de vertu chevaleresque, de générosité, auquel le récit accorde, qui plus est, la palme du martyre. Il nous semble donc qu'on ne prend pas 
beaucoup de risques à voir dans Anseÿs de Gascogne une revanche imaginaire sur le passé et une option idéologique sur l'avenir, minimisant au moins en intention la relative perte d'importance d'un grand feudataire du Nord, le comte de Flandre. Dans la Vengeance Fromondin, on peut tout aussi bien lire une cinglante remise en ordre idéologique du présent, assurant quant à elle la promotion du roi de France.

Pour la Vengeance Fromondin, nombre de données vont à l'encontre de l'esprit même de la Geste, ainsi : outre le caractère positif du roi Pépin, l'absence de mention de la Lorraine, la supériorité numérique de la famille lorraine, pour ne rien dire des contradictions de parentés chez les Boulonnais / Bordelais, du brusque revirement de Ludie, qui change de camp de manière totalement inexplicable à la fin du texte.

Si la cohérence des lignages n'est pas son souci premier, c'est parce que notre poète ne fait qu'emprunter la machinerie épique des Loherains pour parvenir à ses fins. En effet, le grand bénéficiaire du poème, c'est le roi de France. La partialité du poète, qui fait de Pépin un roi à l'autorité redoutable, n'est pas de la même nature que celle qui, dans Garin le Loherain ou dans Gerbert de Metz, donne le beau rôle aux Loherains et le mauvais aux Bordelais. Dans la Vengeance Fromondin, au fond, le poète utilise les personnages de la fiction pour servir son dessein politique et publicitaire au profit de la monarchie capétienne. En ce sens, la Vengeance Fromondin, même si l'on a pu (feindre de) s'y tromper dans un atelier messin dès le dernier tiers du treizième siècle, nous semble une candidate suspecte pour conclure la Geste des Loherains. Comme Anseÿs de Gascogne, poème pour lequel on peut émettre les mêmes réserves, la Vengeance Fromondin n'entre pas dans la logique de la Geste : elle l'instrumentalise.

Alors que Yonnet referme la geste sur elle-même, Anseÿs de Gascogne et la Vengeance Fromondin l'ouvrent sur les enjeux de l'Histoire du XIII ${ }^{\mathrm{e}}$ siècle, la récupèrent pour leurs démonstrations partisanes, la dissolvent dans des préoccupations qui n'étaient pas essentiellement - du moins avec des vues aussi étroites - celles des auteurs de Garin le Loherain et de Gerbert de Metz, même si ces poèmes pouvaient n'être pas dépourvus d'enjeux divers.

En vérité, tout se passe comme si l'auteur de la Vengeance Fromondin voulait, coûte que coûte, en finir avec les Loherains et les Bordelais / Boulonnais, qui ne sont plus d'aucune utilité à son projet de courtisan. Avec le meurtre de Gerbert, il interdit tout prolongement cyclique, d'une part en réglant la vengeance des fils du roi de Gascogne en quatre vers (v. 6630-33), d'autre part en faisant basculer la matière des Loherains dans la Geste de Garin de Monglane (Garin, fils de Gerbert, serait devenu seigneur de Monglane), ce qui rend illégitime un prolongement comme celui d'Anseÿs de Gascogne.

Enfin, par le personnage de Gérin, qui aurait péri à Roncevaux aux côtés de Roland, le poète achève de verrouiller la Geste des Loherains en la reliant au Cycle de Charlemagne. Nommons-le Cycle du Roi : c'est, pour le poète et sa démarche latente de glorification du roi de France contre les seigneurs du Nord, comme le dernier mot de son entreprise. Mais le « Grand Cycle des Loherains » y a perdu son âme...

\section{Une survie paradoxale/Espoir d'un renouveau}

Sur l'importance du Cycle dans la littérature du XIII ${ }^{e}$ siècle, on ne peut avoir aucun doute : les Loherains ont connu un succès tout à fait extraordinaire, non seulement en France - on devrait dire dans le domaine francophone, pour tenir compte des frontières 
du royaume de France à cette époque -, mais encore dans le domaine d'oc et dans le Nord-Ouest de l'Europe.

Il nous est parvenu l'intégralité ou un état fragmentaire d'une cinquantaine de manuscrits français en vers, sans compter ceux qu'on peut légitimement penser perdus, mais dont on a la trace dans divers inventaires anciens. Il est à noter que la plupart des témoins en vers qui nous sont aujourd'hui accessibles datent du XIII ${ }^{\mathrm{e}}$ siècle ou de la première moitié du XIV ${ }^{e}$. Chacune des chansons du Cycle, sauf Yonnet (perdu) et la Vengeance Fromondin ${ }^{48}$, nous a été transmise, si l'on additionne manuscrits intègres et fragments, par un nombre de témoins important: Hervis par cinq témoins ${ }^{49}$, Garin par vingt-neuf, Gerbert par trente-trois, Anseÿs par cinq.

Mais le succès du Cycle se constate aussi, outre par l'identification de remaniements majeurs déjà évoqués, qui confirment sa vitalité (INT, QS notamment) ${ }^{50}$, par le fait que les Loherains ont connu trois mises en prose :

- celle qui se lit dans le manuscrit Arsenal 3346 : elle paraît dater de la fin du XIVe siècle ou de la première moitié $d u X^{e}$; son origine est à peu près impossible à localiser ${ }^{51}$. C'est un travail de bonne tenue, qui abrège considérablement les textes retenus (Gari Gerbert - Anseÿs).

- celle qu'on cite sous le nom de David Aubert, quelle que soit la part réelle qu'il y ait prise ; elle a dû être copiée dans les années 1463-1465, à la Cour de Philippe le Bon, duc de Bourgogne ${ }^{52}$. C'est un travail parfaitement structuré, dont la phrase n'est pas toujours exempte de lourdeurs (Gari-Gerbert - Anseÿs).

- celle que le Messin Philippe de Vigneulles tira en 1514-1515 de manuscrits apparentés au groupe lorrain et aujourd'hui perdus. Dans l'esprit de son auteur, elle est toute dédiée à la gloire de Metz, mais il faut reconnaître que ce que l'on perd en légèreté, on le gagne souvent en précision par rapport aux autres proses; elle ignore Anseÿs de Gascogne et la Vengeance Fromondin, mais elle est la seule prose à connaître, outre Garin/ Gerbert, les textes liminaires, les plus lorrains (Hervis et Yonnet, dont elle est, en outre, notre seul témoin intégral) $)^{53}$.

Succès assuré donc jusqu'au début du XVIe siècle. Sans faire état de rencontres dans lesquelles on ignore, en définitive, quel poète a copié sur l'autre, on doit encore signaler que la Mort Begon, par exemple, a sans doute inspiré, dans le domaine d'oc, le récit de la mort du duc Buevon dans Daurel et Beton; on ne peut, de surcroît, que voir le héros central de notre Geste dans le "Garin le Lieurant" qui, sous le règne de Charlemagne et aux côtés de Guillaume au Court Nez, lutte contre les Sarrasins en Provence, dans le Roman d'Arles (vers 1375) ${ }^{54}$; au Nord, le récit de la chasse au sanglier monstrueux dans Perceforest paraît redevable à l'épisode correspondant des Loherains ${ }^{55}$; de même, certains emprunts à notre Cycle ont été depuis longtemps repérés ici ou là, ainsi, dans Ogier le Danois, qui recycle plusieurs centaines de vers de Gerbert ${ }^{56}$. On signalera aussi Jehan de Lanson (premier tiers du XIII ${ }^{e}$ siècle), le fabliau des Deux bordeors ribaus $\left(\mathrm{XIII}^{2}\right)$, Berte au grans piés d'Adenet le Roi, ouvrages qui font état de leur connaissance de la Geste des Loherains ${ }^{57}$. Au XV siècle, François Villon évoque ensemble Berthe au grand pied, Béatrix et Alix, trois figures féminines qui ne forment un groupe que dans Hervis (respectivement nièce, femme, et mère du héros) ${ }^{58}$.

41 Les chroniqueurs n'ont pas dédaigné, eux non plus, la matière lorraine, qu'ils ont souvent considérée comme un document historique; qu'il nous suffise d'évoquer la Chronographia de Gui de Bazoches (seconde moitié du XII ${ }^{\mathrm{e}}$ siècle), le Chronicon d'Aubri des Trois-Fontaines (première moitié du XIII ${ }^{\mathrm{e}}$ siècle), la Chronique métrique de Philippe 
Mousket (avant 1243), pour les plus anciens, et d'ajouter - qu'on en possède un témoignage direct ou non, que les attributions soient authentiques ou non - Jean d'Outremeuse, Hugues de Toul, Jacques de Guyse, Baudouin d'Avesnes, Symphorien Champier, Édouard de Boulay, Jean Bouchet, Richard de Wassebourg... et quelques autres qui, à l'occasion, ont utilisé les Loherains comme source, avec plus ou moins de précautions ${ }^{59}$.

On pourrait prolonger la liste dans le temps, en citant quelques auteurs qui, ici ou là, mentionnent notre Cycle ou certains de ses héros; ainsi, on rappellera l'intérêt que le magistrat Claude Fauchet $(\dagger 1602)$ trouvait à la lecture de Garin (dont il possédait un manuscrit, l'actuel BNF fr $1442=P$ ), les références diverses faites à notre matière dans les ouvrages de DuCange ${ }^{60}$, Du Chesne, Martin Meurisse, Dom Calmet... Nous ne prétendons pas à l'exhaustivité.

Le constat est clair: les Loherains ont laissé de multiples traces, pendant des siècles, dans la littérature de langue française, chez les chroniqueurs et historiens (en français et en latin). Mais il faut encore prendre en compte l'essaimage du Cycle (ou d'une partie seulement de celui-ci) en dehors du domaine francophone, à travers l'Europe du NordOuest.

Ainsi, nos Loherains sont à la base des Lorreinen néerlandais. En vérité, le Roman der Lorreinen (on l'appelle Roman, parce qu'en néerlandais il n'existe pas de mot pour traduire chanson de geste), semble se composer de trois grandes parties, la première seule dérivant à coup sûr de notre Garin (pour le modèle suivi par les Lorreinen, on peut exclure ACIORT, qui ignorent l'abbé Lietri). Les deux autres parties devaient, d'après l'annonce initiale, amener le récit jusqu'au règne de l'empereur Frédéric. Il est vrai que les Lorreinen ne nous sont parvenus qu'à l'état fragmentaire et que, d'un autre côté, rien ne peut nous assurer que toute la matière épique française ait été conservée ${ }^{61}$.

Qu'il y ait eu parallèlement pénétration de la zone d'influence flamande par nos Loherains, on s'en convaincra encore par le petit poème assonancé qui se lit dans le manuscrit BNF fr 12615: ce texte, où la préposition van remplace volontiers la préposition française de, montre que nos héros tenaient, au XIII siècle, une bonne place dans la matière épique, puisque Gerbert, Gérin, Fromont de Lens et Fromondin y sont mis sur le même plan que Guillaume d'Orange, Charlemagne et Pépin :

Assés l'avés oït van Gerbert, van Gerin

Van Willaume d'Orenge qui vait le chief haiclin,

Van conte de Bouloigne, van conte Hoillequin

Et van Fromont de Lens, van son fils Fromondin,

Van Karlemaine d'Ais, van son pere Pepin...62

Pour terminer sur la diffusion des Loherains hors du domaine francophone, contentonsnous de mentionner encore deux "preuves", plus ou moins assurées, il faut en convenir :

- un énigmatique Livre de Englys del forster et del sangler, livre en anglais parlant d'un forestier et d'un sanglier, ouvrage dans lequel on a voulu voir une version anglaise de la Mort Begon ${ }^{63}$.

- le nom de Lohengrin, dans le Parzival de Wolfram von Eschenbach; Lohengrin représenterait une altération de Loherain Garin, mais l'affaire n'est pas tirée au clair, d'autant qu'il pourrait y avoir une source commune plus ancienne ${ }^{64}$.

En vérité, la grande énigme, ce serait de comprendre pourquoi, après avoir été présents pendant plus de cinq siècles - au grand jour ou de manière plus souterraine dans la 
culture des lettrés -, les Loherains n'ont pas eu, malgré leur passage à la prose, les honneurs de l'imprimé à l'époque où Galien le Restoré, Garin de Monglane, Ogier le Danois et autres Renaut de Montauban, connaissaient plusieurs éditions.

Lassitude du public? Hésitation sur le statut de la matière dont on ne savait plus si elle était littéraire (d'imagination et de divertissement) ou historique (d'étude) ? Volonté de la culture officielle d'effacer le souvenir de textes dans lesquels la monarchie ne donne pas, et c'est peu dire, la meilleure image d'elle-même ? Changement de goût qui relègue les romans de chevalerie parmi les «vieilleries» (mais pourquoi notre Cycle précisément ?) et les ouvrages "gothiques"? On ne peut trancher, mais toutes ces explications, et d'autres encore, probablement, pourraient peut-être rendre compte de l'oubli partiel de nos Loherains à la fin de l'Ancien Régime.

Quoi qu'il en soit, l'intérêt que le romantisme va trouver au Moyen Âge explique la renaissance du Cycle dès 1833. Mais c'est une fausse renaissance, car les Loherains vont d'abord être occultés par la place prise, dès 1840, par le Roland d'Oxford dans les études consacrées à la littérature médiévale. En outre, il y avait alors tant à faire que ce qu'on peut appeler "l'école française" travaillant sur les Loherains, après avoir publié partiellement Garin le Loherain (Paulin Paris, Édélestand du Méril), piétinera et ne produira plus rien en matière d'édition, si ce n'est un projet avorté concernant $\mathrm{Hervis}^{65}$. Toutefois, la première étude d'ensemble de la tradition des Loherains est française, due à François Bonnardot ${ }^{66}$. Si les limites de son travail ont souvent été soulignées, il a eu l'insigne mérite de tenter, pour la première fois, une mise en ordre de la tradition manuscrite complexe alors disponible.

Parallèlement, en Allemagne, après la guerre de 1870, autour notamment du jeune professeur Edmund Stengel ${ }^{67}$, se mit en place une solide équipe de romanistes, qui se consacra dès lors très largement à la matière des Loherains. Il s'ensuivit une floraison d'articles, d'études, d'éditions partielles et de thèses outre-Rhin, sous la plume de W. Benary, O. Böckel, H. Hub, K. Jahn, W. Vietor, et Stengel lui-même ${ }^{68}$. Il semblerait que la guerre de 1870 et le caractère difficile de certains médiévistes, tant en France qu'outre-Rhin, aient rapidement compromis la collaboration entre "l'école française" et "l'école allemande" ${ }^{69}$. De toute façon, Stengel devint aveugle en 1903 et les travaux qu'il dirigeait se tarirent rapidement.

Un peu plus tard, à partir de 1931, à la Columbia University, une "école américaine" se constitua autour du Professeur Pauline Taylor, avec le projet de publier l'intégralité du Cycle des Loherains. On doit à cette équipe : une excellente édition (partielle) de Garin le Loheren (Josephine E. Vallerie 1947), une édition de la fin de Garin et de l'intégralité de Gerbert (Pauline Taylor 1952), l'une et l'autre malheureusement fondées sur A, peu représentatif de l'ensemble de la tradition manuscrite; une édition insuffisante de la Vengeance Fromondin (Simon R. Mitchneck 1935) et une édition d'Anseÿs (Herman J. Green 1939), où le texte est souvent mal compris, reposant, de surcroît, sur le plus atypique des témoins (qui écourte le poème originel de près de treize mille vers). Nous ne sommes pas sûr que le projet initial de l'équipe de la Columbia University ait été complètement accompli ${ }^{70}$, mais il faut reconnaître que l'impulsion donnée par Pauline Taylor a permis les travaux, souvent riches et convaincants, de Jindrich Zezula et, plus largement, de Ruth Parmly, Russel K. Bowman notamment, qui ont cerné avec une précision satisfaisante la place de nos textes dans l'ensemble de la littérature épique française. Plus récemment, Catherine M. Jones s'est attelée à l'étude de Hervis et de quelques points concernant d'autres poèmes de la Geste. 

pas s'être passé grand-chose autour des Loherains avant la fin des années soixante, avant les travaux de Joël H. Grisward; puis ont paru l'adaptation en français moderne de Hervis (Philippe Walter 1984), les traductions de Garin et de Gerbert (Bernard Guidot 1986 et 1988). En 1990, un premier grand colloque français faisait le point sur l'état des travaux consacrés à la Geste des Loherains, colloque organisé à Nanterre, par le Professeur François Suard. Dans les actes de cette manifestation, Bernard Guidot propose une première synthèse de grande ampleur, «L'extension cyclique de la geste des Lorrains: abandons, résurgences, irradiation $»^{71}$, qui développe, dans une perspective différente, ce que nous tentons de faire ici.

On constate qu'après ce colloque, on a assisté à une nouvelle éclosion de publications consacrées aux Loherains, dont nous dressons l'inventaire dans le complément bibliographique qui clôt cette étude. Une mention toute spéciale doit être faite de l'édition de Garin le Loherenc, parue dans une collection française (CFMA, Honoré Champion), et procurée par une collègue américaine (Anne Iker-Gittleman, 1995-96). Le regain d'intérêt des chercheurs pour les Loherains se traduit d'une manière éclatante : la Bibliographie dressée par Bernard Guidot en 1990 comportait 150 entrées (éditions, traductions, ouvrages, articles, comptes rendus) pour une période de cent cinquantecinq années, notre Complément bibliographique en comporte lui aussi 150 pour une période de quatorze années seulement. Il est vrai que les publications universitaires de tous ordres ont eu tendance à voir leur nombre exploser dans les trente dernières années : on doit donc se réjouir que les Loherains n'aient pas laissé passer cette occasion de revenir au premier plan des études littéraires médiévales.

Pour conclure, nous nous contenterons de souligner les difficultés et retards rencontrés pour la transmission du Cycle des Loherains jusqu'à nous, difficultés et retards liés à l'Histoire des deux derniers siècles, bien évidemment, mais aussi et surtout à la complexité de la tradition manuscrite elle-même - nos prédécesseurs allemands n'ont pas abouti à une synthèse cohérente, et nos collègues américains y ont en partie renoncé $^{72}$. Les poèmes de la Geste des Loherains proprement dite et de ses expansions plus ou moins légitimes, au moins du point de vue de l'esprit initial, ce qui ne remet nullement en cause leur valeur intrinsèque - ne constituent pas des œuvres de lecture facile et rapide. Nul doute, cependant, que la moisson ne soit encore très prometteuse, pour qui acceptera d'entrer dans le cercle magique et d'y prendre ses quartiers suffisamment longtemps.

\section{BIBLIOGRAPHIE}

Figurent ici toutes les publications consacrées en priorité aux Loherains, dont nous avons trouvé trace notamment dans le Bulletin de la Société Internationale Rencesvals depuis 1990. Notre Complément, qui ne retient pas toutes les publications où les Loherains ne sont que cités de manière très secondaire, vise à prolonger la Bibliographie donnée par Bernard Guidot dans les

Cahiers de recherches médiévales, 12 | 2005 
Actes du Colloque tenu sur la Geste des Loherains à Nanterre en 1990, Littérales, 10, Nanterre-ParisX 1992, p. 257-275.

Éditions / thèses

Bulté Maggy, Édition des 4400 premiers vers de la version longue de Gerbert d'après le manuscrit W, avec choix de variantes dans tous les autres manuscrits, thèse en cours.

DAQUIN Cécile, Édition synoptique des 4000 derniers vers de Garin le Loherain dans IN, thèse en cours.

HERBIN Jean-Charles, Hervis de Mes, TLF, 414, Droz, Genève 1992.

- Prose des Loherains. Arsenal 3346, Presses Universitaires de Valenciennes, « Parcours » 1995.

- Le début de la Geste des Loherains dans le ms. L-II-14 de Turin (= T), soit les 2300 premiers vers de Garin le Loherain, in Première Journée Valenciennoise de Médiévistique (3 avril 1998), Actes publiés dans Lez Valenciennes, 25, 1999, Presses Universitaires de Valenciennes, p. 131-223.

- Vengeance Fromondin, en cours de publication à la SATF.

- Yonnet de Metz, édition d'après les témoins en prose (en cours).

ION Despina, La parenté dans Garin le Loheren et Gerbert de Mez. Étude littéraire, linguistique et anthropologique, Thèse de doctorat Nouveau Régime, préparée sous la direction du Professeur Bernard Guidot (Nancy-II, 2000), présentée dans Perspectives médiévales, 28, 2002, p. 75-79.

IKER-GITTLEMAN Anne, Garin le Loherain, CFMA, 117-119, H. Champion, Paris 1996-97.

NAUDET Valérie, Guerin le Loherain, édition critique et commentaire de la prose de David Aubert extraite des Histoires de Charles Martel (manuscrit 7 de la Bibliothèque Royale de Belgique), Publications de l'Université de Provence, 2005.

TRIAUD Annie, Anseÿs de Gascogne, thèse en cours.

ARTICLES/COMMUNICATIONS

AUCLAIR Mathias, « Le preux et le saint. Garin le Lorrain et saint Gengoult, ancêtre des ducs de Lorraine », in Romania, 117, 1999, p. 245-257.

BAUMGARTNER Emmanuelle et HARF-LANCNER Laurence, « Raoul de Cambrai : l'impossible révolte ", Collection Unichamp n 83, Champion, Paris 1999.

BARBERO Alessandro, "Les institutions et leur fonctionnement dans l'épopée ", in L'épopée romane au Moyen Âge et aux temps modernes, Actes du XIV Congrès de la Société Rencesvals (1997), Fridericiana Editrice Universitaria, Napoli 2001, tome I, p. 141-161.

BENNETT Philip E., «Le refus d'aide : déni de justice », in Actes du XI Congrès International de la Société Rencesvals (1988), Memorias de la Real Academia de Buenas Letras de Barcelona, 21, 1990, p. 53-64.

BOUILLOT Carine, «Aux antipodes du beau geste : le geste laid et inconvenant dans la littérature des XII ${ }^{\mathrm{e}}$ et XIII ${ }^{\mathrm{e}}$ siècles ", in Le Beau et le Laid, Publications du CUER MA, Senefiance,43, Aix-enProvence 2000, p. 47-56.

BOYER Agustin, « Geography as a linking-Device in the Poema de mio Cid », in Romanic Review, 83-4, 1993, p. 463-474.

BUSBY Keith, « Mise en texte, mise en codex : l'exemple de quelques manuscrits épiques », in Plaist vos oïr bone cançon vallant? Mélanges de Langue et de Littérature Médiévales offerts à François Suard, Collection UL3, Travaux et Recherches, Lille 1999, tome I, p. 141-149.

CAMPBELL Kimberlee A., "The Reiterated Self : Ritual Renewal and Narrative Cycles ", in The Development of Narrative Cycles in the Chansons de Geste and the Arthurian Romances, Amsterdam, 
Oxford, New York, Tokyo 1994, p. 205-209.

- «A note on Crusade and Bourgeois in Hervis de Mes ", in Aspects de l'épopée romane : mentalités, idéologies, intertextualités, Egbert Fornsten, Groningen 1995, p. 217-223.

- «Commemorative formulae in the Geste des Loherens », in Olifant, 19 (1-2), 1994-1995, p. 101-112.

CARERI Maria, « Codici facsimilati e tradizione attiva nella Geste des Loherains », in Romania, 119, 2001, p. 323-356.

CAVANAUGH Susan H., "The identification of a lost English analogue of the "Death of Begon", episode from the Old French epic Garin le Loherain ", in Medium Aevum, LVII-1, 1988, p. 64-67.

CLAMOTE CARRETO Carlos, « Dérision, division, déviation. Incidences poétiques de l'imaginaire marchand sur quelques chansons de geste des XII ${ }^{\mathrm{e}}$ et XIII ${ }^{\mathrm{e}}$ siècles ", in L'épopée romane, Actes du $\mathrm{XV}^{\mathrm{e}}$ Congrès International de la Société Rencesvals, Université de Poitiers (2000), Centre d'Études Supérieures de Civilisation Médiévale, 2002, tome I, p. 265-280.

COMBARIEU DU GRÉS Micheline de, «Bégon le guerrier » in La Geste des Lorrains, Littérales,10, Nanterre-Paris-X 1992, p. 161-188.

- «Les "amis charnels" Garin et Bègue dans Garin le Loherain », in Histoire et Société. Mélanges offerts à Georges Duby, Publications de l'Université de Provence, Aix-en-Provence 1992, p. 125-139.

- « La dernière flèche. Étude sur la mort de Bégon de Belin dans Garin le Lorrain », in Lorraine vivante. Hommage à Jean Lanher, Presses Universitaires de Nancy, 1993, p. 49-57.

- « Le Bruit et la Fureur (sur la violence anarchique dans Anseÿs de Mes) », in La violence dans le monde médiéval, Publications du CUER MA, Senefiance, 36, Aix-en-Provence 1994, p. 121-147. - « Le héros épique peut-il être un héros burlesque ? ", in Burlesque et dérision dans les épopées de l'Occident médiéval, Besançon 1995, p. 25-48.

DUFOUR Louis, « Louis VI, roi de France (1108-1137), à la lumière des actes royaux et des sources narratives ", in Comptes rendus de l'Académie des Inscriptions, avril-juin 1990, p. 456-482.

ESKENAZI André, « Cheval et destrier dans Gerbert de Metz », in La Geste des Lorrains, Littérales,10, Nanterre-Paris-X 1992, p. 115-136.

GRISWARD Joël H., « La cuisine et la guerre : aspects de la fonction guerrière dans la Geste des Loherains ", in L'épopée romane, Actes du XV Congrès International de la Société Rencesvals, Université de Poitiers (2000), Centre d'Études Supérieures de Civilisation Médiévale, 2002, tome I, p. 549-565.

GROSSEL Marie-Geneviève, "Ces "chroniqueurs à l'oreille épique” : remarques sur l'utilisation de la geste chez Philippe Mousket et Aubri des Trois-Fontaines ", in "Ce nous dist li escris... che est la verité ». Études de littérature médiévale offertes à André Moisan par ses collègues et amis, Publications du CUER MA, Senefiance,45, Aix-en-Provence 2000, p. 91-112.

GUIDOT Bernard, "Fixité et dérive dans le Cycle des Lorrains ", in Actes du XI ${ }^{e}$ Congrès International de la Société Rencesvals (1988), Memorias de la Real Academia de Buenas Letras de Barcelona, 21, 1990, p. 321-330.

- «L'extension cyclique de la geste des Lorrains : abandons, résurgences, irradiation ", in La Geste des Lorrains, Littérales,10, Nanterre - Paris-X 1992, p. 15-47.

- «Un dangereux Lucifer bordelais : Bernard de Naisil », in « Et c'est la fin pour quoy sommes ensemble ». Hommage à Jean Dufournet (Littérature, Histoire et Langue du Moyen Âge), H. Champion, Paris 1993, tome II, p. 695-704.

- «Une reine au visage rayonnant : Blanchefleur dans Garin le Loherain », in Lorraine vivante. Hommage à Jean Lanher, Presses Universitaires de Nancy, 1993, p. 77-84.

- «"Enfances", chevalerie et bourgeoisie : idéologie et pratique dans Hervis de Mes ", in Aspects du classicisme et de la spiritualité. Mélanges en l'honneur de Jacques Hennequin, Klincksieck, Paris 1996, 
p. 533-551.

- «Le cadre narratif de Renaut de Montauban : un espace de l'imaginaire », in Tangram. Mélanges

offerts à Claude Wanquet, Publications de l'IUFM de La Réunion, 1996, p. 143-152.

- «Les "enfances" de Vivien ont-elles un caractère romanesque ? ", in Enfances "romanesques", PRIS-MA, XII-2, juillet-décembre 1996, p. 167-186.

- «Constitution de Cycles épiques : étude de quelques jalons ", in Sommes et Cycles (XII ${ }^{e}-X^{2}{ }^{e}$ siècles, Université Catholique de Lyon, 2000, p. 25-47.

HEINTZE Michael, König, Held und Sippe. Untersuchungen zur Chanson de geste des 13. und 14. Jahrhunderts und ihrer Zyklenbildung, Studia Romanica, 76, Carl Winter Verlag, Heidelberg 1991.

HERBIN Jean-Charles, « La "mise en prouse" de la Geste des Loherains par Philippe de Vigneulles : une (re)trouvaille », in Romania, 109, 1988, p. 562-565.

- « Le tournoi de Senlis dans Hervis de Mes », in La Geste des Lorrains, Littérales, 10, Nanterre-Paris-X 1992, p. 71-85.

- «L'arrière-plan socio-économique de Hervis de Mes », in Heldensage-Heldenlied-Heldenepos, Wodan, 12, Jahrbücher der Reineke-Gesellschaft, Série 4, Band 2, juillet 1992, p. 137-149.

- « Approches de la mise en prose de la Geste des Loherains par Philippe de Vigneulles ", in Romania, 113, 1992-1995, p. 466-504.

- « La mise en prose de la Geste des Loherains dans le manuscrit Arsenal 3346 », in Traduction, transcription, adaptation au Moyen Âge. Bien dire et bien aprandre, 13, 1996, Revue du Centre d'Études médiévales et dialectales de Lille-III, p. 237-256.

- «Châteaux et forteresses dans la Geste des Loherains ", in Le château médiéval et la guerre dans l'Europe du Nord-Ouest. Mutations/Adaptations, Revue du Nord, Hors série, Collection Art et Archéologie $\mathrm{n}^{\circ}$ 5, 1998, p. 9-19.

- «Les “enfances romanesques" deHervis de Metz », in Enfances "romanesques”, PRIS-MA,XII-1, janvier-juin 1996, p. 27-37.

- "Itinéraires dans la chanson de Garin le Loherain ", in La géographie dans les textes médiévaux, Wodan-Série 3, vol. 38, Reineke Verlag 1996, p. 59-82.

- « Activité poétique et ménestrels à Valenciennes aux XIV et $\mathrm{XV}^{\mathrm{e}}$ siècles », in Valenciennes aux $X I V^{e}$ et $X V^{e}$ siècles, sous la direction de Ludovic Nys et d'Alain Salamagne, Presses Universitaires de Valenciennes, 1996, p. 297-316.

- « Guichardet/Begonnet : une rencontre entre le Cycle de Guillaume et la Geste des Loherains? », in Medioevo Romanzo, 21, 1997, p. 276-295.

- «L'enchanteur Tulles dans la chanson d'Anseÿs de Metz », in Magie et illusion au Moyen Âge, Publications du CUER MA, Senefiance, 42, Aix-en-Provence 1999, p. 209-232.

- « Notice du manuscrit $h$ de la Prose des Loherains par Philippe de Vigneulles », in Romania, 117, 1999, p. 218-244.

- «L'épisode du crâne de Fromont dans Gerbert de Metz », in Plaist vos oïr bone cançon vallant? Mélanges de Langue et de Littérature Médiévales offerts à François Suard, Collection UL3, Travaux et Recherches, Lille 1999, tome I, p. 407-422.

- «Châlons-en-Champagne dans la Geste des Loherains », in Châlons au XII ${ }^{e}$ siècle, Imprimerie du Conseil Général de la Marne, avril 1999, p. 29-53.

- «Sarrasins et chrétiens dans la Geste des Loherains ", in La Chrétienté au péril sarrasin, Publications du CUER MA, Senefiance,46, Aix-en-Provence 2000, p. 51-76.

- «Raoul de Cambrai et la Geste des Loherains ", in "Raoul de Cambrai" entre l'épique et le romanesque, Littérales, 25, Nanterre-Paris-X 1999, p. 79-101.

- « La Champagne dans Hervis de Metz et Garin le Loherain », in Mémoires de Champagne, D. Guéniot, Langres 2001, tome III, p. 163-181.

- « Lumineuse Beatrix : un aspect de la stratégie narrative du poète de Hervis de Metz », in Clarté. Essais sur la lumière, PRIS-MA, XVII-2, n 34, juillet-décembre 2001, p. 209-216. 
- « Anseÿs de Gascogne et la Flandre », in Le picard d'hier et d'aujourd'hui. Bien dire et bien aprandre,21, octobre 2003, p. 207-228.

- «La rédactionC de la Chevalerie Vivien », in Richesses Médiévales du Nord et du Hainaut, Presses Universitaires de Valenciennes, 2002, p. 11-23.

- « L'Histoire otage des chansons de geste ou l'inverse ? Le cas d'Anseÿs de Gascogne et de la Vengeance Fromondin ", in Le Nord de la France entre épopée et chronique, Artois Presses Université 2005, p. 239-265.

- «Yonnet de Metz. Mise en prose d'un poème perdu », Communication lors de la Journée d'Étude «Les mises en prose », organisée à Lille-III le 19 mars 2004, à paraître.

- «Auberi le Bourguignon, personnage de Garin le Loherain », Communication au Colloque "L'épopée médiévale et la Bourgogne », organisé à Dijon les 14-15 octobre 2004, à paraître. - « Un "notable clerc" et la médecine médiévale », Contribution aux Mélanges offerts au Professeur Gabriel Bianciotto, à paraître.

- «"Trois fuelles d'erbe a pris entre ses piez". Recherches sur la Mort Begon dans Garin le Loherain », à paraître.

HUGEUX Xavier, «La caractérisation des personnages secondaires dans Gerbert de Metz », in La Geste des Lorrains, Littérales, 10, Nanterre - Paris X 1992, p. 201-216.

- «La reine et le clan messin dans Gerbert de Metz », in Lorraine vivante - Hommage à Jean Lanher, Presses Universitaires de Nancy, 1993, p. 103-107.

ION Despina, « Remarques sur les relations de parenté dans Garin le Loherain et Gerbert de Metz », in L'épopée : mythe, histoire, société, Littérales, 19, 1996, p. 133-151.

- «Banquet, conflit et fidélité dans Garin le Loherain », in Banquets et manières de table au Moyen Âge, Publications du CUER MA, Senefiance, 38, Aix-en-Provence 1996, p. 303-318.

- «L'esprit généalogique comme stratégie littéraire dans le Prologue de Garin le Loherain », in Plaist vos oür bone cançon vallant? Mélanges de Langue et de Littérature Médiévales offerts à François Suard, Collection UL3, Travaux et Recherches, Lille 1999, tome I, p. 445-454.

- « Politique matrimoniale et stratégies narratives dans Garin le Loheren », in L'épopée romane au Moyen Âge et aux temps modernes, Actes du XIV Congrès International de la Société Rencesvals (1997), Fridericiana Editrice Universitaria, Napoli, 2001, tome I, p. 247-265.

- « La parenté comme outil narratif dans Garin le Loherain », in L'épopée romane, Actes du XV Congrès International de la Société Rencesvals, Université de Poitiers (2000), Centre d'Études Supérieures de Civilisation Médiévale, 2002, tome I, p. 567-576.

JONES Catherine M., " "Se je fusse hons" : les guerrières dans Anseÿs de Mes ", in Charlemagne in the North: Proceedings of the Twelfth International Conference of the Société Rencesvals, Edinburgh 1993, p. 291-297.

- « L'enlèvement dans Garin le Loherain et Hervis de Metz », in Plaist vos oïr bone cançon vallant? Mélanges de Langue et de Littérature Médiévales offerts à François Suard, Collection UL3, Travaux et Recherches, Lille 1999, tome I, p. 455-463.

- «"Modernizing" the Epic : Philippe de Vigneulles », in Echoes of the Epic. Studies in honor of Gerard J. Brault, Birmingham, Alabama, Summa Publications Inc. 1998, p. 115-132.

- « Autour du "nouveau langage" : la Geste des Loherains aux XVI ${ }^{\mathrm{e}}$ et XIX siècles ", in L'épopée romane au Moyen Âge et aux temps modernes, Actes du XIV ${ }^{\mathrm{e}}$ Congrès International de la Société Rencesvals (1997), Fridericiana Editrice Universitaria, Napoli, 2001, p. 693-704.

KRAUß Henning, «"Noblesse utérine oblige doublement” - Zur Ätiologie des Loherains im Hervis de Mes, in Literatur - Geschichte und Verstehen » - Festschrift für Ulrich Mölk 60. Geburtstag, Winter, Heidelberg, 1997, p. 239-250.

LABBÉ Alain, « Le cadre architectural de Garin le Lorrain : régression du modèle palatin et prédominance du décor castral », in La Geste des Lorrains, Littérales, 10, Nanterre - Paris X 1992, 
p. 217-242.

- « "Contremont Muese a retorné son vis". Le regard des Lorrains sur la Lorraine dans Garin le Loherain ", in Lorraine vivante. Hommage à Jean Lanher, Presses Universitaires de Nancy, 1993, p. 109-114.

- « Un locus amoenus de la féodalité menacée : la description de Géronville dans Gerbert de Mez », in La description au Moyen Âge, Bien dire et bien aprandre, 11, 1993, p. 239-260.

- «"Vivrez en voz ?" Le corps et la mort dans Garin le Loherain et dans Gerbert de Mez ", in Le corps et ses énigmes au Moyen Âge, Paradigme, Caen 1993, p. 87-120.

- «Un cas particulier de la topographie castrale des chansons de geste : la forteresse maritime », in La Chanson de Geste. Écritures, intertextualités, translations, Littérales, 14, 1994, p. 61-82.

- « La dérision des clercs dans Girart de Roussillon et Garin le Loheren », in Burlesque et dérision dans les épopées de l'Occident médiéval, Besançon 1995, p. 351-378.

- «La poétique des ruines dans quelques chansons de geste », in Littératures, 37, Presses

Universitaires de Toulouse-Le Mirail, 1997, p. 235-259.

- « Guerre sainte et guerre privée dans les chansons de geste : Girart de Roussillon, Garin le Loheren, Gerbert de Mez ", in Littérature et religion au Moyen Age et à la Renaissance, Presses Universitaires de Lyon, Collection «XI-XVI Littérature », 1997, p. 47-64.

- « La Gironde épique : la fascination de l'estuaire dans Gerbert de Metz et Renaut de Montauban », in Nouvelle Revue d'Onomastique, 29-30, 1997, p. 213-224.

- « L'architecture du désastre dans Anseÿs de Mes », in Travaux de Littérature, 12, 1999, p. 215-236.

- «L'autre guerrier : altérité et proximité dans quelques chansons de geste », in L'autre et les encyclopédies, Université de Rennes (Cahiers Diderot 11), 1999, p. 67-92.

- «Quand les saints vivaient parmi nous : sainteté, pouvoir et condamnation du siècle dans Anseÿs de Mes ", in Essais sur la perfection. Le héros et le saint, PRISMA, XVI-1, n 31, janvier-juin 2000, p. 77-100.

- « De quelques cœurs arrachés : horreur et violence dans la geste de la révolte », in L'Horreur au Moyen Âge, Université de Toulouse-II, Éditions universitaires du Sud, Diffusion Champion, 2000, p. 75-96.

- «La chevalerie épique au péril de la mer : la tempête d'Anseÿs de Mes », in Guerres, voyages et quêtes au Moyen Âge. Mélanges offerts à Jean-Claude Faucon, Champion, Paris 2000, p. 219-232.

- " "Li garçonnés petis" : l'enfant et le saint dans Anseÿs de Mes ", in "Ce nous dist li escris... che est la verité ». Études de littérature médiévale offertes à André Moisan par ses collègues et amis, Publications du CUER MA, Senefiance, 45, Aix-en-Provence 2000, p. 123-139.

- «Un épisode plaisant dans Anseÿs de Mes : la nuit d'amour interrompue du comte Baudouin », in "Si a parlé par moult ruiste vertu ». Mélanges de littérature médiévale offerts à Jean Subrenat, Champion, Paris, 2000, p. 313-324.

- «Gerbert et Fromondin, ou le dévoiement de l'hospitalité dans deux épisodes de Gerbert de Metz », in Représentations médiévales de l'hospitalité, Littérales, 27, 2000, p. 49-73.

- « Sous le signe de saint Jacques : routes et itinéraires dans la représentation épique de l'espace ", in L'épopée romane, Actes du XV $\mathrm{XV}^{\mathrm{e}}$ Congrès International de la Société Rencesvals, Université de Poitiers (2000), Centre d'Études Supérieures de Civilisation Médiévale, 2002, tome I, p. 99-118.

LENS Mieke, « Old French Epic Cycles in MS. Turin L.II.14 : the Development of Old French Narrative Cycles and the Transmission of such Cycles into Middle Dutch Epic Poetry ", in The Development of Narrative Cycles in the Chansons de Geste and the Arthurian Romances, Amsterdam, Oxford, New York, Tokyo 1994, p. 127-134.

MARTIN Jean-Pierre, "Oratores et bellatores dans le Cycle des Lorrains », in Actes du XI Congrès International de la Société Rencesvals (1988), Memorias de la Real Academia de Buenas Letras de Barcelona, 22, 1990, p. 25-38. 
- «Croisade et lutte contre les infidèles dans le cycle des barons révoltés », in La Croisade : Réalités et fictions, (Göppinger Arbeiten zur Germanistik, 503), Kümmerle Verlag, Göppingen 1989, p. 157-167.

- «Lire Garin le Loherain hors du manuscrit A », in La Geste des Lorrains, Littérales, 10, NanterreParis-X 1992, p. 89-114.

- « La rencontre d'Ysoré de Boulogne et de Huon de Cambrai dans Garin le Loherain », in Lorraine vivante. Hommage à Jean Lanher, Presses Universitaires de Nancy, 1993, p. 129-135.

- « Notes sur le manuscrit de Bruxelles de Garin le Loherain », in Convergences médiévales. Épopée, lyrique, roman. Mélanges offerts à Madeleine Tyssens, Bibliothèque du Moyen Age 19, De Boeck, Louvain 2000, p. 319-326.

MÉNARD Philippe, «Le rire et le sourire dans les premières chansons de geste des Lorrains (Garin le Lorrain et Gerbert de Metz) », in La Geste des Lorrains, Littérales, 10, Nanterre-Paris-X 1992, p. 139-159.

NAUDET Valérie, «Quand le roi frappe la reine : à partir d'une scène de la Geste des Lorrains ", in Le Geste et les gestes de table au Moyen Âge, Publications du CUER MA, Senefiance, 41, Aix-enProvence 1998, p. 443-459.

- « La Geste des Lorrains de David Aubert. Vogue d'un genre et originalité d'un texte », in L'épopée tardive, Littérales, 22, Nanterre-Paris-X 1998, p. 151-167.

- «Étude du duel judiciaire de Bégon de Belin et Isoré dans Garin le Loherenc », in « Si a parlé par moult ruiste vertu ». Mélanges de littérature médiévale offerts à Jean Subrenat, Champion, Paris 2000, p. 409-417.

- «La Chrétienté au péril sarrasin ? (Les Sarrasins dans la Geste des Loherains) », in La Chrétienté au péril sarrasin, Publications de l'Université de Provence, Senefiance, 46, 2000, p. 161-182.

- « Le tremble et le château. Étude de l'espace dans la version bourguignonne de Guerin le Loherain ", in Le Nord de la France entre épopée et chronique, Artois Presses Université 2005, p. 267-283.

PARISSE Michel, «Garin le Loherain dans l'histoire de son temps », in La Geste des Lorrains, Littérales, 10, Nanterre-Paris-X 1992, p. 51-63.

PICHERIT Jean-Louis, « L'hôtellerie, les hôteliers et hôtelières dans quelques œuvres de la fin du Moyen Âge ", in Le Moyen Age, 108, 2002, p. 301-332.

ROQUES Gilles, «Commentaires sur quelques régionalismes lexicaux dans le Roman de Troie de Beneeit de Sainte More ", in Troie au Moyen Âge, Bien dire et bien aprandre, 10, p. 157-170.

SUARD François, Présentation des Actes du Colloque La Geste des Lorrains, Littérales, 10, NanterreParis-X 1992, p. 5-11.

- «Le Cycle des Lorrains », in The Development of Narrative Cycles in the Chansons de Geste and the Arthurian Romances, Amsterdam, Oxford, New York, Tokyo 1994, p. 219-220.

- «L'épopée médiévale et la Picardie », in Perspectives médiévales, 20, 1994, p. 68-79.

- « La Chrétienté au péril de l'invasion sarrasine », in La Chrétienté au péril sarrasin, Publications de l'Université de Provence, Senefiance, 46, 2000, p. 231-248.

- Avant-Propos des Actes du Colloque La Chanson de Geste. Écritures, intertextualités, translations, Littérales, 14, 1994, p. 5-6.

- Conclusions des Actes du Colloque L'épopée : mythe, histoire, société, Littérales, 19, 1996, p. 123-130.

SUBRENAT Jean, « Bauche li cors comte, ermite et martyr », in La Geste des Lorrains, Littérales, 10, Nanterre-Paris-X 1992, p. 189-200.

VALLECALLE Jean-Claude, « Du merveilleux au rationnel : Galopin dans Garin le Loherain », in Lorraine vivante. Hommage à Jean Lanher, Presses Universitaires de Nancy, 1993, p. 223-227. 
VAN DER HAVE Jakob B., « Les Loherains à l'étranger », in Au carrefour des routes d'Europe : la chanson de geste, Publications du CUER MA, Senefiance, 21, Aix-en-Provence 1987, p. 1089-1095. - « Les Lorrains néerlandais : continuation ou innovation », in La Geste des Lorrains, Littérales, 10, Nanterre-Paris-X 1992, p. 65-70.

- Roman der Lorreinen : de fragmenten en het geheel, Uitgverij Scriptum, Schiedam 1990 (Sommaire en français aux p. 174-178).

WALTER Philippe, « Tout commence par des chansons... (Intertextualités lotharingiennes) », in Styles et valeurs. Pour une histoire de l'art littéraire au Moyen Âge, Textes réunis par Daniel Poirion, SEDES, Paris 1990, p. 187-209.

WEILL Isabelle, «Les syntagmes du cœur dans la Geste des Lorrains ", in Le "cuer" au Moyen Âge. Réalité et "senefiance", Publications du CUER MA, Senefiance, 30, Aix-en-Provence 1991, p. 461-470. - « Blanchart ou le destrier, le guerrier et le royaume dans Auberi le Bourguignon ", in Le cheval dans le monde médiéval, Publications du CUER MA, Senefiance, 32, Aix-en-Provence 1992, p. 573-584. - « Les structures de parenté dans Garin et Gerbert », in La Geste des Lorrains, Littérales, 10, Nanterre-Paris-X 1992, p. 243-253.

Comptes rendus

AILES M., C. R. d'Anne Iker-Gittleman, Garin le Loherenc, in Medium Aevum, 67, 1998, p. 153-154.

BELLON Roger, C. R. d'Anne Iker-Gittleman, Garin le Loherenc, in Perspectives médiévales, 23, 1997, p. 55-57.

- C. R. de Jean-Charles Herbin, Prose des Loherains (Ms. Arsenal 3346), in Perspectives Médiévales, 25, 1999, p. 129.

BRANDSMA F., C. R. de J. B. Van der Have, Roman der Lorreinen : fragmenten en het geheel, in Millenium. Tijdschrift voor Middeleeuwse studies, 5, 1991, p. 81-83.

COLBY-HALL A. M., C. R. de Catherine M. Jones, The Noble Merchant: Problems of Genre and Lineage in Hervis de Mes, in Speculum, 70-1, 1995, p. 158-160.

DEMBOWSKI P. F., C. R. d'Anne Iker-Gittleman, Garin le Loherenc, in Speculum, 74-1, 1999, p. 190-191.

DE WILDE P., C. R. de Jean-Charles Herbin, La mise en prose de la Geste des Loherains dans le manuscrit Arsenal 3346, in Scriptorium, 51, 1997, p. 52-53.

GIER A., C. R. de Jean-Charles Herbin, Hervis de Mes, in Zeitschrift für Romanische Philologie, 111, 1995, p. 280-281.

GUIDOT Bernard, C. R. d'Anne Iker-Gittleman, Garin le Loherenc, in Cahiers de Civilisation Médiévale, 41,1998 , p. 40-43.

HERBIN Jean-Charles, Présentation de l'édition de Hervis de Mes, in Perspectives Médiévales, 17, 1991, p. 115-118.

- C. R. d'Anne Iker-Gittleman, Garin le Loherenc, in Romania,118, 2000, p. 542-556.

HUGEUX Xavier, C. R. de Bernard Guidot, Gerbert : chanson de geste du XIII ${ }^{e}$ siècle traduite en français moderne, in Cahiers de Civilisation Médiévale, 36, 1993, p. 96-97.

JONES Catherine M., C. R. de Bernard Guidot et Jean Lanher, Gerbert : chanson de geste $\mathrm{du}$ XIII ${ }^{e}$ siècle traduite en français moderne, in Olifant, 14, 1989, p. 212-216.

KIBLER W. W., C. R. de Jean-Charles Herbin, Hervis de Mes, in Romance Philology, 48-3, 1994-1995, p. 302-306.

- C. R. d'Anne Iker-Gittleman, Garin le Loherenc, in Romance Philology, 52, 1998, p. 164-169. 
MOORMAN B. D., C. R. de Bernard Guidot et Jean Lanher, Gerbert : chanson de geste du XIII ${ }^{e}$ siècle traduite en français moderne, in Olifant, 13, 1988, p. 115-118.

ROQUES Gilles, C. R. de Jean-Charles Herbin, Prose des Loherains (Ms. Arsenal 3346), in Revue de Linguistique Romane, 60, p. 311.

- C. R. d'Anne Iker-Gittleman, Garin le Loherenc, in Revue de Linguistique Romane, 60, 1996, p. 610.

THIRY-STASSIN M., C. R. d'Alain Labbé, « “Li garçonnés petis” : l'enfant et le saint dans Anseÿs de Mes », in « Ce nous dist li escris... », in Scriptorium, 56, 2002, p. 65.

TILMANS K., C. R. de J. B. Van der Have, Roman der Lorreinen : fragmenten en het geheel, in Bijdragen en Mededelingen betreffende de geschiedenis der Nederlanden, 107, 1992, p. 589.

VAN EMDEN A. G., C. R. de Jean-Charles Herbin, Hervis de Mes, in French Studies, 48, 1994, p. $187-188$.

VARVARO A., C.R. de J. C. Herbin, Prose des Loherains (Ms. Arsenal 3346), in Medioevo Romanzo, 22, 1998, p. 458.

WALTER Philippe, C. R. de Jean-Charles Herbin, Hervis de Mes, in Cahiers de Civilisation médiévale, 37-4, 1994, p. 378-380.

\section{NOTES}

*. Pour cette contribution, qui ne se veut pas une synthèse globale et définitive, nous avons à l'esprit les éditions suivantes: Garin le Loheren, éd. Anne Iker-Gittleman, CFMA, 117-119, H. Champion, Paris 1996-97 ; Gerbert de Mez, éd. Pauline Taylor, Bibliothèque de la Faculté de Philosophie et Lettres de Namur, fascicule XI, Namur-Louvain-Lille 1952; Hervis de Mes, éd. J. C. Herbin, TLF, 414, Droz, Genève 1992; pour la Vengeance Fromondin, nous avons utilisé notre édition en cours de publication à la SATF ; pour Anseÿs de Gascogne, nos propres fichiers et la thèse en cours de Madame Annie Triaud.

1. Cf. Paulin Paris, Li romans de Garin le Loherain, Techener, Paris 1853, tome I, p. XVI.

2. Cf. Édélestand du Méril, La Mort Garin le Loherain, Franck, Paris 1846, p. LI, LVII, LVIII, LIX notamment.

3. Cf. Paulin Paris, "Étude sur les chansons de geste et sur Garin le Loherain », in Le Correspondant, LVIII, 1863, p. 722.

4. Idem, p. 722.

5. Ibidem, p.722-723; il convient de noter que la publication du Recueil des anciens historiens des Gaules et de la France (Dom Bouquet) n'était pas encore terminée à l'époque où Paulin Paris écrivait ces lignes.

6. Ibidem, p. 745, où il désigne Anseÿs comme « la seconde partie de la Geste ».

7. Cf. Paulin Paris, Histoire littéraire de la France, tome XXII, Paris 1895, p. 641 ; il avait, dès 1833, repoussé l'idée que l'ensemble aurait pu, d'après la présence de très grandes initiales dans les manuscrits, s'organiser en six chansons successives, cf. Li romans.., ouvrage cité, p. XIX.

8. Elle paraît acceptée, en effet, par Edmund Stengel, et Pauline Taylor, notamment, ainsi que par leurs émules.

9. Cf. Jean-Charles Herbin, «L'Histoire otage des chansons de geste ou l'inverse ? Le cas d'Anseÿs de Gascogne et de la Vengeance Fromondin", in Le Nord de la France entre épopée et chronique, Artois Presses Université 2005, p. 239-265.

10. Cf. Malcolm Vale, The princely court-Medieval Courts and Cultures in North-West Europe, 1270-1380, University Press, Oxford, Appendix VIII, p. 368. 
11. Le texte en vers d'Yonnet est perdu; mais nous possédons la prose qu'en a tirée Philippe de Vigneulles; nous travaillons actuellement à une édition de cette prose inédite et qui n'a jamais été prise en considération dans la constitution de la Geste des Loherains, dont Yonnet offre pourtant la seule fin appropriée.

12. On pense, notamment, aux variations et contradictions liées aux filles du duc Hervis; cf. Jean-Charles Herbin, « Auberi le Bourguignon, personnage de Garin le Loherain », Communication au Colloque "L'épopée médiévale et la Bourgogne », organisé à Dijon les 14-15 octobre 2004, à paraître.

13. Comme nous l'établissons pour la Vengeance Fromondin dans l'Introduction de l'édition que nous avons actuellement sous presse.

14. Rappelons que Garin nous est parvenu dans vingt et un manuscrits à peu près complets ou suffisamment bien conservés pour qu'on puisse utilement les prendre en compte comme témoins de la tradition (ABCDEFGIJLMNOPQRSTVWX), Garin ayant encore laissé une trace dans huit fragments et Gerbert dans quatorze, ce qui permet de postuler l'existence d'au moins quarantetrois manuscrits aux treizième / quatorzième siècles, chiffre tout à fait extraordinaire, si l'on songe aux sept manuscrits de la Chanson de Roland, pour ne rien dire des témoins uniques, parfois très mutilés, qui nous ont transmis Gormont et Isembard, la Chanson de Guillaume, le Voyage de Charlemagne...

15. Une seule exception, le manuscrit $D$ (= BNF fr 1461), mais c'est un codex composite, ce qui ne prouve, en définitive, pas grand-chose.

16. Cette très grande initiale se trouve, dans les manuscrits CDEFJLMPSW, à la suite du dernier vers du poème de Garin, tel qu'il est donné dans l'édition de Mme Iker-Gittleman; la jointure correspond au vers 2471 de l'édition de Gerbert par Pauline Taylor; sur ce découpage aberrant, voir, entre autres, Jean-Pierre Martin, «Lire Garin le Loherain hors du manuscrit A », in La Geste des Lorrains, Études réunies et présentées par François Suard, Littérales, 10, Nanterre-Paris-X 1992, p. 89-114, en particulier, les pages 90 et suivantes.

17. Au moins un des manuscrits parvenus commence avec Garin II ( $X=B N F$ fr 2179, groupe lorrain); un autre présente une coupure explicite entre Garin I et Garin II ( $=$ Dijon 528); les manuscrits CDGNOQRSVWXZ ${ }^{8}$ présentent une très grande initiale pour le vers Un jor fu Begue el chastel de Belin, qui ouvre Garin II ; dans N, il y a même, entre Garin I et II, un petit quatrain qui indique le nom du copiste et du propriétaire du manuscrit et paraît terminer le texte du premier modèle suivi ; pour davantage de précisions, voir Jean-Charles Herbin, «Approches de la mise en prose de la Geste des Loherains par Philippe de Vigneulles ", in Romania, 113, 1992-1995, p. 466-504.

18. Nous dirigeons, depuis 2001, la thèse de Maggy Bulté, qui éditera prochainement les 4400 premiers vers de la version longue de Gerbert d'après le manuscrit $W$, avec choix de variantes dans tous les autres manuscrits donnant cette version.

19. À titre indicatif, le corpus fourni par tous les témoins et fragments des Loherains - dont nous possédons intégralement copie sous forme de microfilms - représente, pour l'ensemble des poèmes, plus de 650000 vers, auxquels il faut joindre, pour des comparaisons souvent productives, les trois proses qui nous sont parvenues, dont deux peuvent paraître démesurées (celle de David Aubert occupe près de 1380 feuillets, celle de Philippe de Vigneulles compte, sous format informatique, 2200000 caractères...).

20. Cf. Edward Le Glay, « La mort de Bégon de Belin », in Archives historiques et littéraires du Nord de la France, tome IV, 1835, p. 193-231.

21. Cf. Paulin Paris : «Si j'ose même exprimer toute ma pensée, je ne connais rien de plus beau dans la poésie épique que les derniers instants de Bégon et le récit des vengeances que sa mort occasionne ", in Li romans..., ouvrage cité, tome I, p. XIX.

22. On se reportera, entre autres, à J. C. Herbin, «Itinéraires dans la chanson de Garin le Loherain ", in La géographie dans les textes médiévaux, Wodan, Série 3, vol. 38, Reineke Verlag 1996, 
p. 59-82 ; - " “Trois fuelles d'erbe a pris entre ses piez”. Recherches sur la Mort Begon dans Garin le Loherain ", parution prévue dans la revue Le Moyen Âge (Louvain).

23. À noter que le microtoponyme de Gademont est transmis par plusieurs témoins, même approximativement (cf. J. C. Herbin, "Itinéraires... », article cité, p. 64), alors que Raismes, pris pour un nom commun par les scribes (et par les éditeurs modernes), est perdu comme toponyme, sauf dans 0 .

24. Cf. Régine Le Jan, Famille et pouvoir dans le monde franc (VII ${ }^{e}-X^{e}$ siècle), Publications de la Sorbonne, Paris 1995, en particulier les pages 255-256 et le tableau de la page 442.

25. Sur les groupes et familles de manuscrits que l'on peut déterminer à l'intérieur de la Vulgate, voir Jean-Pierre Martin, article cité, p.96; Anne Iker-Gittleman, Le style épique dans Garin le Loherain, Droz, Genève 1967, p. 12-20, et Garin le Loherenc, éd. citée, tome I, p. 17-24 et 32-42.

26. Nous rejetons la forme Grais de Mont, donnée par $F$ seul, qui ne correspond à rien sur le terrain.

27. La trace de cette évolution apparaît quand on compare, notamment, la Vie de saint Colomban (VIII ${ }^{\mathrm{e}}$ siècle) et les textes de la fondation de l'Abbaye d'Hasnon (1079). Nous reprendrons cette question un jour prochain.

28. L'information peut paraître quelque peu gratuite; toutefois, à faible distance de Valenciennes, à l'Ouest, dans l'ancien royaume de Lothaire donc, existent deux localités nommées Wargnies, toponyme remontant à un ancien Gariniacas, ce qui ne s'invente pas...

29. Sur cette question, on consultera la synthèse proposée par Bernard M. Pohoryles, "Sur la datation de Garin le Loherain et Gerbert de Mez ", in Atti del XIV. Congresso de linguistica et filologia romanza, Napoli 1981, p. 333-342.

30. Cf. en particulier, Ferdinand Lot, "L'élément historique de Garin le Lorrain", in Études d'Histoire du Moyen Age dédiées à Gabriel Monod, Paris 1896, p. 201-220, en particulier les pages 204-210 ; voir aussi, plus récemment, la synthèse proposée par Jindrich Zezula, in La Geste des Loherains et les chroniqueurs : le fond historique et la datation de Garin le Loherain et de Gerbert de Mez, Ph. D. de l'Université de New York, 1968, en particulier les tableaux des pages 216-220.

31. Cf. Jindrich Zezula, La Geste des Loherains.., ouvrage cité, p. 217-220.

32. Il est à noter que la famille NT, qui remanie le Prologue de Garin, élimine le personnage de l'archevêque de Reims; (I)NT paraissent liés à l'abbaye de Saint-Amand, cf. notre étude : «Le début de la Geste des Loherains dans le manuscrit L-II-14 de Turin (T)», in Première journée valenciennoise de médiévistique, Lez Valenciennes $n^{\circ} 25$, Presses Universitaires de Valenciennes, 1999, p. 131-223, en particulier les pages 135-139; si le remanieur à l'origine de la famille INT a réécrit le Prologue sans le personnage de l'archevêque pour complaire à quelque grand personnage, on peut signaler une rencontre bien intéressante : en 1192, l'abbé de Saint-Nicaise de Reims devient abbé de Saint-Amand: a-t-il amené avec lui un clerc disposé à rendre service à la mémoire de l'archevêque Henri († 1175) si mal traité dans le Prologue de Garin?

33. Le seul personnage de ce nom dont nous ayons trouvé la trace au Moyen Âge, un certain Johannes de Flagi cité dans l'obituaire du Prieuré de Saint-Martin-des-Champs, paraît ne pas convenir, car il est cité pour le XIV ${ }^{\mathrm{e}}$ siècle, cf. Obituaires de la Province de Sens, tome I, publié par Auguste Molinier, Imprimerie Nationale, Paris 1902, p. 423 (toutefois, ce personnage pourrait avoir été inscrit dans l'obituaire « à une date assez éloignée de [sa] mort », cf. p. 420).

34. Cf. Félix Lecoy, « Sur Gerbert de Metz : lieux et date », in Romania, 77, 1956, p. 435.

35. Cf. Jean-Charles Herbin, "L'épisode du crâne de Fromont dans Gerbert de Metz ", in Plaist vos oïr bone cançon vallant? Mélanges de Langue et de Littérature Médiévales offerts à François Suard, Textes réunis par. D. Boutet, M.-M. Castellani, F. Ferrand, A. Petit, Collection UL3, Travaux et Recherches, Lille 1999, tome I, p. 407-422, plus particulièrement les pages 417-418; la suite de nos recherches, toujours en cours, nous amène à penser que Gerbert est probablement le plus "aquitain" des poèmes des Loherains.

36. Cf. Félix Lecoy, article cité, p. 430-431. 
37. On notera que le groupe dit « ancien » (ABCO, Cf. Jean-Pierre Martin, article cité, p. 96) ne se repère vraiment, d'après nos relevés (c'est-à-dire dans les initiatives d'ouverture de laisses nouvelles), que pour les 2900 premiers vers de Gerbert ou, si l'on préfère, pour le début de la version longue de ce poème.

38. Cf. notamment, François Bonnardot, "Essai de classement des manuscrits des Loherains ", in Romania, 3, 1874, p. 195-262, en particulier les pages 253-257.

39. C'est, au fond, ce que François Bonnardot appelait la « rédaction primitive s'arrêtant avec Gerbert ", article cité, p. 255.

40. Cf. Jean-Charles Herbin, "Le tournoi de Senlis dans Hervis de Mes ", Littérales, 10, 1992, Nanterre-Paris-X, p. 71-85.

41. Voire un peu plus haut encore, si le document de l'Abbaye de Beaupré, qui donne un paysan nommé Garin comme fils d'une certaine Béatrix, a quelque chose à voir avec notre poème, dont il attesterait le succès dès les dernières années du XII ${ }^{\mathrm{e}}$ siècle, $\mathrm{cf}$. Ch. Edmond Perrin, Recherches sur la seigneurie rurale en Lorraine (d'après les plus anciens censiers, IX ${ }^{\mathrm{e}}$-XII ${ }^{\mathrm{e}}$ siècles), Paris 1935, Megariotis Reprints, Genève (s. d.), p. 738 : «... Warinus et mater ejus Beatrix... ».

42. Le texte de Hervis, qui ne donne qu'une fille à Hervis et à Béatrix, et non sept comme le fait Garin, pourrait même avoir conservé une donnée d'un état de Garin antérieur à celui que nous a transmis la Vulgate; sur cette question, voir Jean-Charles Herbin, «Auberi le Bourguignon... », article cité, à paraître.

43. Noter, toutefois, que $E$ est un codex composite, dont les deux constituants ne sont pas de la même main et peuvent dater de décennies différentes de la seconde moitié du XIII ${ }^{\mathrm{e}}$ siècle, ce qui rendrait leur juxtaposition plus accidentelle qu'intentionnelle, au moins du point de vue littéraire.

44. Cf. Hervis de Mes, éd. citée, Annexe XXIV, p. 471-520.

45. Nous avons sur cette prose un article en cours de publication, article dans lequel nous puisons l'essentiel de la présente mise au point.

46. Nous reprenons ici très largement nos travaux antérieurs, cf. notre Introduction à la Vengeance Fromondin, (sous presse) et «L'Histoire otage des chansons de geste... », article cité, en particulier les pages de conclusion, dont nous reproduisons ici l'essentiel.

47. «Anseÿs de Gascogne et la Flandre », Communication présentée au Colloque des 4-5-6 octobre 2001 «Le picard d'hier et d'aujourd'hui », organisé par le Centre d'Études Dialectales de l'Université de Lille III ; actes parus dans Bien dire et bien aprandre,21, 2003, p. 207-228.

48. Mais la Vengeance Fromondin est une expansion de Gerbert avant d'être une chanson autonome, même s'il est clair que le texte de cette expansion fonctionne plus sur lui-même qu'à l'intérieur du Cycle dont il ne partage ni ne poursuit l'esprit.

49. Auxquels on ajoutera, même s'il ne s'agit pas d'une œuvre de premier plan, la Chronique messine rimée, datée de la fin $d u X X^{e}$ ou du début $d u X^{e}$ siècle, qui connaît les deux mille premiers vers de Hervis (qu'elle résume en soixante-douze vers); cf. par exemple, Austrasie (Revue de Metz et de Lorraine), $4^{\mathrm{e}}$ volume, 1856, p. 237-238.

50. Il est vrai que la date des innovations est délicate à préciser, ainsi que l'impact de la mise en forme matérielle des témoins INT et $Q S$, qui datent tous, semble-t-il, du dernier tiers du $\mathrm{XIII}^{\mathrm{e}}$ siècle ou du premier tiers du XIV ${ }^{\mathrm{e}}$; sur la fin particulière de Garin dans IN, nous dirigeons, depuis 2000, la thèse de Cécile Daquin, qui éditera les quatre mille derniers vers de ce poème dans chacun des témoins $I N$.

51. Nous l'avons publiée en 1995 aux Presses Universitaires de Valenciennes; voir aussi notre étude, "La mise en prose de la Geste des Loherains dans le manuscrit Arsenal 3346 ", Communication au Colloque International de Lille-III, 22-24 septembre 1994, in Bien dire et bien aprandre, 13, 1996, Revue du Centre d'Études médiévales et dialectales de Lille-III, p. 237-256.

52. La partie concernant Garin vient d'être publiée, cf. Valérie Naudet, David Aubert - Guerin le Loherain, Publications de l'Université de Provence, 2005. 
53. Sur cette prose, on peut consulter Karl Jahn, Philipp de Vigneulle's Yonnet de Mes und sein Verhältnis zur Redaktion N des Romans Anseïs de Mes, Dissertation, Greifswald 1903 ; Jean-Charles Herbin, "La "mise en prouse" de la Geste des Loherains par Philippe de Vigneulles: une (re)trouvaille », in Romania, 109, 1988, p. 562-565 ; - «Approches de la mise en prose de la Geste des Loherains par Philippe de Vigneulles", in Romania 113, 1992-1995, p. 466-504 ; - « Notice du manuscrit $\boldsymbol{h}$ de la Prose des Loherains par Philippe de Vigneulles ", in Romania, 117, 1999, p. 218-244: - «Yonnet de Metz. Mise en prose d'un poème perdu », Communication lors de la Journée d'Étude «Les mises en prose ", organisée à Lille-III le 19 mars 2004 ; actes à paraître.

54. Cf. Le Roman d'Arles, éd. C. Chabaneau, in Revue des Langues Romanes, XXXII, 1888, p. 473-542, en particulier les pages 505-507, lignes 962, 998, 1007 (?), ainsi que les notes correspondantes.

55. Cf. Jean-Charles Herbin, «Un avatar de la Mort Begon dans Perceforest: réécriture ou réminiscence? », Image et mémoire du Hainaut médiéval,Presses Universitaires de Valenciennes, 2004, p.193-206.

56. Cf. Emil Heuser, "Die Chanson des Loherains eine Quelle der Chevalerie Ogier ", in Ausgaben und Abhandlungen aus dem Gebiete der romanischen Philologie, LXXII, 1886, Anhang, p.68-87; Barbara Demarco-Schurfranz, "La Chevalerie Ogier : verbatim borrowing from the Geste des Loherains ", in Romance Philology, XXX, 1977, p.470-474; on évoquera simplement ici le Guillaume de Dole, qui prétend citer une laisse de Gerbert dont on ne trouve la trace dans aucun manuscrit et dont on peut se demander s'il ne s'agit pas d'un pastiche.

57. Voir aussi Russell K. Bowman, The Connections of the Geste des Loherains with other French Epics and Mediaeval genres, New York 1940.

58. Cf. Louis Thuasne, Euvres de François Villon, Picard, Paris 1923, tome II, p. 151-152, 655 et suivantes ; Philippe Ménard, " "Berte au grant pié, Bietris, Alis" ou la résurgence de la culture épique dans la "Ballade des dames du temps jadis" ", in Romania, 102, 1981, p. 114-129.

59. Sur cet aspect de la postérité des Loherains, on se reportera à l'étude fort riche de Jindrich Zezula, ouvrage cité, p. 8-146; il faut reconnaître que les emprunts plus ou moins repérables, parfois de seconde main, font qu'il est difficile au chercheur moderne de s'y retrouver dans les textes des chroniqueurs cités; le mérite est d'autant plus grand pour notre collègue américain d'avoir ouvert la voie et de l'avoir utilement balisée.

60. Cf. Charles Du Cange, Histoire de l'état de la ville d'Amiens et de ses comtes..., Amiens 1840, p. 170-173 (où il propose d'identifier le Hardré de nos Loherains à Walderic, comte de Soissons).

61. Sur cette question, voir Jakob B. Van der Have, "Les Loherains à l'étranger ", in Au carrefour des routes d'Europe : la chanson de geste, Senefiance, 21, CUER MA 1987, p. 1089-1095 ; - « Les Lorrains néerlandais : continuation ou innovation », in La Geste des Lorrains, Littérales, 10, p. 65-70; et plus largement, sur l'ensemble des Lorreinen, du même, Roman der Lorreinen : de fragmenten en het geheel, Uitgverij Scriptum, Schiedam 1990 (Sommaire en français aux p. 174-178).

62. Citation d'après Gaston Raynaud, "La Mesnie Hellequin ", in Études romanes dédiées à Gaston Paris, Paris 1891, p. 51-68, en particulier la page 59.

63. Cf. Susan H. Cavanaugh, "The identification of a lost English analogue of the "Death of Begon", episode from the Old French epic Garin le Loherain ", in Medium Aevum, LVII-1, 1988, p. 64-67: le livre ici en cause est cité (dans un inventaire anglais de 1388) juste après un exemplaire du Roman de Meis, dans lequel on doit pouvoir voir un témoin de Garin le Loherain ; voir aussi Anne Iker-Gittleman, éd. citée, tome I, p. 27.

64. Cf. Erich von Richthofen, «Le substrat hispano-portugais de la légende de Loherenc et de l'Arthur d'Algarve ", in Anuario de Estudios medievales (Barcelona), tome II, 1965, p. 525-530.

65. Cf. l'annonce insérée par François Bonnardot et E. de Bouteillier dans Romania, 7, 1878, p. 350 ; on doit aussi signaler ici l'édition d'extraits et l'étude de l'épisode de Senlis de Hervis par l'historien François Bourquelot, dans Études sur les foires de Champagne, Mémoires présentés par divers savants à l'Académie des Inscriptions et des Belles-Lettres de l'Institut Impérial de France, Deuxième Série, tome V, Paris 1865, p. 109-129. 
66. Pour la fin de la présente étude, nous citons les éditeurs et les critiques qui se sont attachés à la Geste des Loherains sans fournir les références de leurs travaux : jusqu'en 1990, elles figurent dans la Bibliographie complète donnée par Bernard Guidot dans les Actes du colloque de Nanterre (Littérales, 10, p. 257-275) ; après 1990, dans le Complément bibliographique qui termine la présente étude.

67. On peut, toutefois, citer pour mémoire un pionnier allemand qui a travaillé en même temps que les premiers spécialistes français de notre Cycle, Fr. J. Mone, "Werin von Lothringen », in Anzeiger für Kunde der teutschen Vorzeit, Karlsruhe, IV-1835, p. 346-347, V-1836, p. 360-362, VIII-1838, p.408-411; du même, Untersuchungen zur Geschichte der teutschen Heldensagen, Quedlinburg und Leipzig 1836, en particulier les pages 192-292, qui donnent de longs extraits de Garin et de Gerbert d'après le manuscrit $Q$ (Bruxelles).

68. On trouvera la liste de ces travaux dans la Bibliographie dressée par Bernard Guidot dans Littérales, 10, p. 257-275.

69. Les Archives de l'Université catholique de Louvain possèdent, dans leur fonds Stengel, au moins un courrier qui laisse penser que l'édition de Hervis avait été, à l'origine, conçue comme un travail franco-allemand ; la rupture de Stengel avec ses collègues français (rupture qui doit dater d'avant 1878, d'où l'annonce insérée alors dans la Romania, cf. plus haut, note 66) amena le savant romaniste à changer son manuscrit de base.

70. Contrairement à la note de Jean Misrahi, "The Loherain Cycle: a project completed", in Traditio, IX, 1953, p. 435-436.

71. Cf. La Geste des Lorrains, Littérales, 10, p. 15-47.

72. Cf. Pauline Taylor, Gerbert de Mez, Bibliothèque de la Faculté de Philosophie et Lettres de Namur, fascicule XI, Namur-Louvain-Lille 1952, p. XXVII.

\section{AUTEUR}

\section{JEAN-CHARLES HERBIN}

Université de Valenciennes et du Hainaut-Cambrésis 